\title{
VECTOR PARAMETERS IN CLASSICAL HYPERBOLIC GEOMETRY
}

\section{DANAIL BREZOV, CLEMENTINA MLADENOVA AND IVAÏLO MLADENOV}

Presented by Ivaïlo M. Mladenov

Abstract. Here we use an extension of Rodrigues' vector parameter construction for pseudo-rotations in order to obtain explicit formulae for the generalized Euler decomposition with arbitrary axes for the structure groups in the classical models of hyperbolic geometry. Although the construction is projected from the universal cover $\operatorname{SU}(1,1) \simeq \mathrm{SL}(2, \mathbb{R})$, most attention is paid to the $2+1$ Minkowski space model, following the close analogy with the Euclidean case, and various decompositions of the restricted Lorentz group $\mathrm{SO}^{+}(2,1)$ are investigated in detail. At the end we propose some possible applications in special relativity and scattering theory.

\section{Contents}

1 Introduction $\quad 21$

2 Projective Construction $\quad 2$

2.1 The Poincaré Disk and Pseudo-quaternions _ . . . . . . . . . . . 22

2.2 The Upper Half Plane Model . . . . . . . . . . . . . . . . . . . . 23

2.3 Rodrigues' Formula and Vector Parametrization . . . . . . . . . . . . 25

2.4 Composition Law for the Vector Parameters . . . . . . . . . . . . . . 27

3 Generalized Euler Decomposition 29

3.1 The Case of Three Axes . . . . . . . . . . . . . . . . . . . . . . 29

3.2 The Case of Two Axes . . . . . . . . . . . . . . . . . 33

3.3 Degenerate Solutions . . . . . . . . . . . . . . . . . 34

3.4 The Trivial Element . . . . . . . . . . . . . . . . . . . . 35

3.5 Divergencies . . . . . . . . . . . . . . . 35

4 Null Vectors and Invariant Axes

4.1 Euler's Fixed Axis Theorem . . . . . . . . . . . . . . . . . . . 37

4.2 The Parabolic Case . . . . . . . . . . . . . . . . . . . . . . . 39

4.3 The Embedding $\mathrm{SO}(2) \subset \mathrm{SO}^{+}(2,1) \ldots \ldots \ldots . \ldots . \ldots 41$

doi: 10.7546/jgsp-30-2013-19-48 19 
5 Wigner Rotation

6 Concluding Remarks

References

46

\section{List of Symbols and Notations}

$$
\begin{aligned}
& \hat{\mathbf{c}}_{1}, \hat{\mathbf{c}}_{2}, \hat{\mathbf{c}}_{3}, \hat{\mathbf{n}} \\
& \text { normalized vectors in } \mathbb{R}^{2,1} \\
& \mathbf{e}_{x}, \mathbf{e}_{y}, \mathbf{e}_{z} \\
& \text { unit vectors along the coordinate axes } \\
& \mathbf{e}_{k}, \tilde{\mathbf{e}}_{k} \\
& z_{k}, \alpha, \beta, \alpha_{k}, \beta_{k} \\
& \Re(z), \Im(z) \\
& \xi, \zeta, \tilde{\xi}, \tilde{\zeta} \\
& \mathbf{c}, \tilde{\mathbf{c}}, \mathbf{c}_{1}, \mathbf{c}_{2}, \mathbf{c}_{3} \\
& \mathbf{x} \otimes(\eta \mathbf{z})^{t} \\
& \times, \curlywedge \\
& (\mathbf{x}, \mathbf{z}), \mathbf{x} \cdot \mathbf{z} \\
& \varepsilon_{i j k} \\
& \mathbf{c}^{\times}, \mathbf{c}^{\curlywedge} \\
& \mathcal{I}, \eta \\
& \mathcal{A}(\mathrm{x})=\mathrm{x}^{\curlywedge} \\
& \mathcal{R}_{h}(\mathbf{c}), \mathcal{R}_{h}\left(\mathbf{c}_{k}\right) \\
& \epsilon=\operatorname{sgn}(\mathbf{c} \cdot \mathbf{c}) \\
& \tau, \tilde{\tau}, \tau_{k} \\
& \varphi, \theta, \phi, \gamma, \psi_{k} \\
& \kappa_{i j}=\hat{\mathbf{c}}_{i} \cdot \hat{\mathbf{c}}_{j}, \quad \epsilon_{i}=\kappa_{i i} \\
& \sigma_{i j}=\hat{\mathbf{c}}_{i} \cdot \mathcal{R}_{h}(\mathbf{c}) \hat{\mathbf{c}}_{j} \\
& \text { bases in } \mathfrak{s u}(1,1) \text { and } \mathfrak{s l}(2, \mathbb{R}) \\
& \text { complex numbers } \\
& \text { real and imaginary part of a complex number } \\
& \text { pseudo-quaternions } \\
& \text { vector parameters of pseudo-rotations } \\
& \text { dyadic product of vectors in } \mathbb{R}^{2,1} \\
& \text { cross product of vectors in } \mathbb{R}^{3} \text { and } \mathbb{R}^{2,1} \\
& \text { dot product of vectors in } \mathbb{R}^{3} \text { and } \mathbb{R}^{2,1} \\
& \text { the Levi-Civita symbol } \\
& \text { the Hodge dual in } \mathbb{R}^{3} \text { and } \mathbb{R}^{2,1} \\
& \text { the identity matrix and the flat metric in } \mathbb{R}^{2,1} \\
& \text { an element of the Lie algebra } \mathfrak{s o}(2,1) \\
& \text { pseudo-rotations in } \mathbb{R}^{2,1} \\
& \text { sign of the pseudo-norm } \\
& \text { scalar parameters } \\
& \text { angles and rapidities } \\
& \text { elements of the Gramm matrix } \\
& \text { matrix entries of } \mathcal{R}_{h}(\mathbf{c}) \\
& \rho_{k}=\hat{\mathbf{n}} \cdot \hat{\mathbf{c}}_{k}, \quad \varepsilon_{i j k} \tilde{\rho}^{k}=\hat{\mathbf{c}}_{i} \curlywedge \hat{\mathbf{c}}_{j} \cdot \hat{\mathbf{n}} \\
& \omega=\hat{\mathbf{c}}_{1} \cdot \hat{\mathbf{c}}_{2} \curlywedge \hat{\mathbf{c}}_{3} \\
& \text { some useful scalar products } \\
& \text { a volume element }
\end{aligned}
$$




\section{Introduction}

Vector decompositions of arbitrary rotations in $\mathbb{R}^{3}$ into pairs or triplets of ortogonal transformations have been studied repeatedly since the time of Euler, who was the first to propose a parametrization of $\mathrm{SO}(3)$ with three angles, later named after him. We can distinguish two powerful algebraic methods to deal with the more general case of nonorthogonal axes. The first one, explicitly relying on Rodrigues' formula, was proposed by Davenport [9] and recently exploited by Piovan and Bullo [27] in a more consistent framework. The second one is based on the vector parameter construction, proposed by Rodrigues [29] and developed in the works of Gibbs [11], who used to call it "vector semitangent of version" (now it is commonly known as Gibbs' or Rodrigues' vector) and Fedorov [10], while two of the present authors are among the first to utilize it in classical mechanics $[23,24]$ and certain quantum mechanical problems [19,20,22]. To our knowledge it was first exploited in the context of Euler decomposition by Wohlhart [34], but the idea was fully developed only recently in [5,21], where analytic solutions based on this construction are presented.

Apart from its merits in terms of clarity and simplicity, vector parameter representation appears to provide most natural parametrization of $\mathrm{SO}(3)$, as it is given by a projection from the universal cover, which has not been sufficiently emphasized by Gibbs and Fedorov. Here we use analogous construction to obtain the generalized Euler parameters for a decomposition of an arbitrary element of the restricted Lorentz group ${ }^{1} \mathrm{SO}^{+}(2,1)$ projecting from its spin cover $\mathrm{SU}(1,1)$. We also write the corresponding expressions in the $\operatorname{SL}(2, \mathbb{R})$ case and thus provide explicit solutions for the classical models of hyperbolic geometry - the Poincaré disk $\Delta$, the upper half-plane $\mathcal{H}^{2}$ and the flat three-dimensional Minkowski space $\mathbb{R}^{2,1}$.

In the following section we introduce pseudo-quaternions [8, 32] (also known as split quaternions) and construct the hyperbolic vector parameter with their help. In Section 3 we derive a simple method to obtain the Euler parameters in the decomposition of a generic pseudo-orthogonal transformation in $2+1$ dimensions with respect to two or three almost arbitrary axes, paying special attention to more specific cases, such as degenerate solutions and divergent parameters. Next we investigate into more detail how parabolic elements and pure rotations behave within the group and finally provide a relevant physical example in Section 5.

\footnotetext{
${ }^{1}$ This is just the connected component of unity in $\mathrm{SO}(2,1)$, also called orthochronous, since its elements preserve the direction of time in $2+1$ dimensional special relativity.
} 


\section{Projective Construction}

In this section we introduce the basic machinery needed for our work - Rodrigues' formula and the vector parameter representation in the hyperbolic case. The starting point is the universal cover $\mathrm{SU}(1,1)$, built in a quaternion fashion, in complete analogy with the Euclidean case. Then, following this analogy further, we project onto $\mathrm{SO}^{+}(2,1)$ where most of the work is being done. We also comment on the corresponding construction on $\mathrm{SL}(2, \mathbb{R})$ using the well-known isomorphism of the Lie groups (see $[12,13])$.

\subsection{The Poincaré Disk and Pseudo-quaternions}

We may choose a basis in $\mathfrak{s u}(1,1)$ in the form

$$
\mathbf{e}_{1}=\left(\begin{array}{ll}
0 & 1 \\
1 & 0
\end{array}\right), \quad \mathbf{e}_{2}=\left(\begin{array}{rr}
0 & \mathrm{i} \\
-\mathrm{i} & 0
\end{array}\right), \quad \mathbf{e}_{3}=\left(\begin{array}{rr}
\mathrm{i} & 0 \\
0 & -\mathrm{i}
\end{array}\right) .
$$

Then, adding the identity element

$$
\mathbf{e}_{0}=\left(\begin{array}{ll}
1 & 0 \\
0 & 1
\end{array}\right)
$$

we easily obtain a realization of the group $\mathrm{SU}(1,1)$ as a submanifold of $\mathbb{R}^{4}$. More precisely, each vector $\zeta \in \mathbb{R}^{4}$ can be expanded as $\zeta=\zeta_{i} \mathbf{e}_{i}, i=0 \ldots 3$ in the above basis (summation over repeated indices is always assumed in the text, if not pointed otherwise). Using complex coordinates $\alpha=\zeta_{0}+\mathrm{i} \zeta_{3}$ and $\beta=\zeta_{1}+\mathrm{i} \zeta_{2}$ we may write

$$
\zeta=\left(\begin{array}{cc}
\alpha & \beta \\
\bar{\beta} & \bar{\alpha}
\end{array}\right)
$$

which is invertible as long as $|\alpha|^{2}-|\beta|^{2} \neq 0$ (i.e., for any non-isotropic vector $\left.\zeta \in \mathbb{R}^{2,2}\right)$ and if we choose $|\alpha|^{2}-|\beta|^{2}=1$, we obtain an element of $\mathrm{SU}(1,1)$ with inverse, equal to

$$
\zeta^{-1}=\left(\begin{array}{cc}
\bar{\alpha} & -\beta \\
-\bar{\beta} & \alpha
\end{array}\right)
$$

In this way we easily identify $\mathrm{SU}(1,1)$ with the hyperboloid (or quasi-sphere)

$$
\zeta_{0}^{2}-\zeta_{1}^{2}-\zeta_{2}^{2}+\zeta_{3}^{2}=1
$$

For more details on the subject of quaternions and hypercomplex numbers we refer to $[8,17,32]$ and [33] for some of their application in mechanics. 


\subsection{The Upper Half Plane Model}

It is well known that $\operatorname{SL}(2, \mathbb{R})$ acts as a structure group in the upper half-plane $\mathcal{H}^{2}=\{z \in \mathbb{C} ; \Im z>0\}$ via linear fractional transformations

$$
\mathrm{SL}(2, \mathbb{R}) \ni A=\left(\begin{array}{ll}
a & b \\
c & d
\end{array}\right): \quad z \longrightarrow A(z)=\frac{a z+b}{c z+d} \in \mathcal{H}^{2}
$$

and since the action is only determined up to a scalar factor, we think of it as a representation of the projective group $\operatorname{PSL}(2, \mathbb{R})$, also called the Möbius group of $\mathcal{H}^{2}$. Its Lie algebra is certainly the same - we may choose a basis in the space of $2 \times 2$ traceless real matrices $\mathfrak{s l}(2, \mathbb{R})$ in the form

$$
\tilde{\mathbf{e}}_{1}=\left(\begin{array}{ll}
0 & 1 \\
1 & 0
\end{array}\right), \quad \tilde{\mathbf{e}}_{2}=\left(\begin{array}{rr}
1 & 0 \\
0 & -1
\end{array}\right), \quad \tilde{\mathbf{e}}_{3}=\left(\begin{array}{rr}
0 & 1 \\
-1 & 0
\end{array}\right)
$$

and use it to expand vectors in $\mathbb{R}^{2,1}$ as we did before. Moreover, we may add the identity element

$$
\tilde{\mathbf{e}}_{0}=\left(\begin{array}{ll}
1 & 0 \\
0 & 1
\end{array}\right)
$$

and thus represent the group itself. Actually, instead of rebuilding the whole construction from scratch, we give an explicit morphism between the two groups which will help us adapt the results obtained above without any trouble. We use the well-known geometric correspondence

$$
\mathcal{U}: \mathcal{H}^{2} \rightarrow \Delta, \quad z \rightarrow \mathcal{U}(z):=\mathrm{i} \frac{z-\mathrm{i}}{z+\mathrm{i}}
$$

that maps the upper half-plane to the unit disk and which can be extended to their groups of isometries. Its matrix

$$
\mathcal{U}=\frac{1}{\sqrt{2}}\left(\begin{array}{rr}
1 & -\mathrm{i} \\
-\mathrm{i} & 1
\end{array}\right)
$$

is unitary (with this particular normalization) and it is not hard to see that it maps the basis $\left\{\tilde{\mathbf{e}}_{k}\right\}$ to $\left\{\mathbf{e}_{k}\right\}$ and vice versa via conjugation

$$
\mathbf{e}_{k}=\mathcal{U} \tilde{\mathbf{e}}_{k} \mathcal{U}^{\dagger}, \quad \tilde{\mathbf{e}}_{k}=\mathcal{U}^{\dagger} \mathbf{e}_{k} \mathcal{U}
$$

which means that the Lie algebras are isomorphic and the same holds for the groups as well. Hence, all results for $\mathrm{SU}(1,1)$ can be directly applied to $\operatorname{SL}(2, \mathbb{R})$ and vice versa. For a more advanced study on hyperbolic geometry we refer to $[30,31]$. 
There are three classes of elements in $\operatorname{PSL}(2, \mathbb{R})$ and we have an elementary criterion to distinguish between them: for each matrix $\zeta \in \mathrm{SL}(2, \mathbb{R})$ the corresponding linear-fractional transformation is

1. hyperbolic if $|\operatorname{Tr} \zeta|>2$

2. elliptic if $|\operatorname{Tr} \zeta|<2$

3. parabolic if $|\operatorname{Tr} \zeta|=2$

so if we expand the vector part of $\zeta$ over the basis specified in (4) we end up with

$$
\zeta=\left(\begin{array}{ll}
\zeta_{0}+\zeta_{2} & \zeta_{1}+\zeta_{3} \\
\zeta_{1}-\zeta_{3} & \zeta_{0}-\zeta_{2}
\end{array}\right)
$$

and by this construction it becomes evident that the above conditions concern only its scalar part. In the parabolic case for example, we have $\zeta_{0}=1$ and since $\operatorname{det} \zeta=1$ this yields the equation of the light cone

$$
\zeta_{1}^{2}+\zeta_{2}^{2}-\zeta_{3}^{2}=0 .
$$

Similarly hyperbolic transformations are associated with space-like vectors and the elliptic ones - with time-like vectors, the above quadratic form being positive in the former while negative in the latter case. It is easy to see for example, that Möbius transformations, corresponding to translations $z \rightarrow z+\alpha$

$$
\mathcal{T}_{p}(\alpha)=\left(\begin{array}{cc}
1 & \alpha \\
0 & 1
\end{array}\right)
$$

are parabolic and direct comparison with (8) gives $\zeta_{2}=0$ and $\zeta_{1}=\zeta_{3}=\frac{\alpha}{2}$.

As for the elliptic and hyperbolic transformations, by the exponential map, we have two typical representatives in the form

$$
\mathcal{T}_{e}(\varphi)=\left(\begin{array}{rr}
\cos \varphi & \sin \varphi \\
-\sin \varphi & \cos \varphi
\end{array}\right), \quad \mathcal{T}_{h}(\tau)=\left(\begin{array}{ll}
\mathrm{e}^{\tau} & 0 \\
0 & \mathrm{e}^{-\tau}
\end{array}\right) .
$$

Moreover, each element $\mathcal{T}$ of $\operatorname{PSL}(2, \mathbb{R})$ can be written as (see [1])

$$
\mathcal{T}=\mathcal{T}_{e} \mathcal{T}_{h} \mathcal{T}_{p}
$$

Note that if we restrict our considerations to integer coordinates on the light cone, the components of $\zeta$ form a Pythagorean triple, and thus we obtain a representation of the Pythagorean triples in the modular group (see [16] for details). 


\subsection{Rodrigues' Formula and Vector Parametrization}

Any vector $\mathbf{x} \in \mathbb{R}^{2,1}$ expanded in the basis (1) can be mapped in the algebra $\mathfrak{s u}(1,1)$ as follows

$$
\mathbf{x} \rightarrow \mathbf{X}=\left(\begin{array}{cc}
\mathrm{i} x_{3} & x_{1}+\mathrm{i} x_{2} \\
x_{1}-\mathrm{i} x_{2} & -\mathrm{i} x_{3}
\end{array}\right)
$$

while its pseudo-Euclidean norm is given by

$$
\mathbf{x} \cdot \mathbf{x}=-\operatorname{det} \mathbf{X}=x_{1}^{2}+x_{2}^{2}-x_{3}^{2} .
$$

Then we may easily construct a norm preserving automorphism, using the adjoint action of the group in its algebra

$$
\operatorname{Ad}_{\zeta}: \mathbf{X} \rightarrow \zeta \mathbf{X} \zeta^{-1}
$$

The Cartesian coordinates of $\mathrm{x}$ are then being transformed by a pseudo-orthogonal matrix $\mathcal{R}_{h}(\zeta)$ which is easily shown to have the form

$$
\mathcal{R}_{h}(\zeta)=\left(\begin{array}{ccc}
1-2 \zeta_{1}^{2} & -2\left(\zeta_{1} \zeta_{2}+\zeta_{0} \zeta_{3}\right) & 2\left(\zeta_{1} \zeta_{3}+\zeta_{0} \zeta_{2}\right) \\
2\left(\zeta_{0} \zeta_{3}-\zeta_{1} \zeta_{2}\right) & 1-2 \zeta_{2}^{2} & 2\left(\zeta_{2} \zeta_{3}-\zeta_{0} \zeta_{1}\right) \\
2\left(\zeta_{0} \zeta_{2}-\zeta_{1} \zeta_{3}\right) & -2\left(\zeta_{2} \zeta_{3}+\zeta_{0} \zeta_{1}\right) & 1-2 \zeta_{3}^{2}
\end{array}\right) .
$$

Then we may separate the scalar and vector part of the pseudo-quaternion

$$
\zeta=\left(\zeta_{0}, \zeta\right), \quad \zeta \in \mathbb{R}^{2,1}
$$

which allows to write down the above matrix invariantly

$$
\mathcal{R}_{h}(\zeta)=\mathcal{I}-2 \zeta \otimes(\eta \zeta)^{t}+2 \zeta_{0} \zeta^{\curlywedge}
$$

In the above formula $\mathcal{I}$ is the $3 \times 3$ identity operator, $\eta=\operatorname{diag}(1,1,-1)$ is the flat metric tensor in $\mathbb{R}^{2,1}, \mathcal{P}_{\boldsymbol{\zeta}}=\boldsymbol{\zeta} \otimes(\eta \boldsymbol{\zeta})^{t}$, which can be explicitly written as $\mathcal{P}_{\zeta_{j}}^{i}=\eta_{j k} \zeta^{i} \zeta^{k}$, is a quasi-projector along $\zeta$, obtained by the usual tensor or dyadic product of vectors and $\zeta^{\curlywedge}=\eta \boldsymbol{\zeta}^{\times}$(written in components as $\boldsymbol{\zeta}_{j}^{\curlywedge i}=\eta^{i k} \varepsilon_{k l j} \zeta^{l}$ ) is the tensor, associated with $\zeta$ by the Hodge duality

$$
\zeta^{\curlywedge} \xi=\zeta \curlywedge \xi=\eta \zeta \times \xi .
$$

Note that a covariant definition of the tensor entities we work with allows for using $\times$ instead of $\curlywedge$ and $\boldsymbol{\zeta} \otimes \boldsymbol{\zeta}$ for $\boldsymbol{\zeta} \otimes(\eta \boldsymbol{\zeta})^{t}$ - switching from upper to lower indices and vice versa then naturally involves the metric $\eta$. However, we chose to make our expressions as explicit as possible here and in order to avoid confusion, introduce 
the unnecessary otherwise notation $\curlywedge$ and $\zeta \otimes(\eta \zeta)^{t}$.

Now, since from (3) and (10) we have $\zeta_{0}^{2}-\zeta^{2}=1$, where $\zeta^{2}=\boldsymbol{\zeta} \cdot \boldsymbol{\zeta}$, as long as $\boldsymbol{\zeta}$ is space-like $\left(\zeta^{2}>0\right)$ we may introduce a unit vector

$$
\hat{\mathbf{n}} \in \mathbb{R}^{2,1}, \quad \hat{\mathbf{n}} \cdot \hat{\mathbf{n}}=1
$$

to parameterize $\mathcal{R}_{h}(\zeta)$ by writing

$$
\zeta_{0}=\cosh \frac{\varphi}{2}, \quad \zeta=\sinh \frac{\varphi}{2} \hat{\mathbf{n}}, \quad \varphi \in \mathbb{R}
$$

and then with the help of some basic hyperbolic trigonometry (12) becomes

$$
\mathcal{R}_{h}(\hat{\mathbf{n}}, \varphi)=\cosh \varphi \mathcal{I}+(1-\cosh \varphi) \hat{\mathbf{n}} \otimes(\eta \hat{\mathbf{n}})^{t}+\sinh \varphi \hat{\mathbf{n}}^{\curlywedge}
$$

which we may refer to as the hyperbolic Rodrigues' formula and the pseudoangular parameter $\varphi$ is usually called rapidity. Furthermore, we may introduce the hyperbolic vector parameter $\mathbf{c} \in \mathbb{R P}^{3}$ in a projective manner

$$
\mathbf{c}=\frac{\zeta}{\zeta_{0}}=\tanh \frac{\varphi}{2} \hat{\mathbf{n}}=\tau \hat{\mathbf{n}}
$$

where the $\tau \in \mathbb{R}$ is referred to as the scalar parameter of the pseudo-rotation. Hyperbolic Euler substitution then leads to the alternative representation

$$
\mathcal{R}_{h}(\mathbf{c})=\frac{\left(1+\mathbf{c}^{2}\right) \mathcal{I}-2 \mathbf{c} \otimes(\eta \mathbf{c})^{t}+2 \mathbf{c}^{\curlywedge}}{1-\mathbf{c}^{2}} .
$$

Note that our hyperbolic metric allows to have vectors with negative scalar squares, called time-like vectors. If $\zeta$ is such a vector $\left(\zeta^{2}<0\right)$, it obviously cannot be proportional to a unit one (at least not with a real coefficient), but we may introduce a quasi-unit time-like vector

$$
\hat{\mathbf{n}} \in \mathbb{R}^{2,1}, \quad \hat{\mathbf{n}} \cdot \hat{\mathbf{n}}=-1
$$

and then the natural parametrization is

$$
\mathbf{c}=\frac{\zeta}{\zeta_{0}}=\tan \frac{\varphi}{2} \hat{\mathbf{n}}=\tau \hat{\mathbf{n}}
$$

or

$$
\zeta_{0}=\cos \frac{\varphi}{2}, \quad \zeta=\sin \frac{\varphi}{2} \hat{\mathbf{n}}, \quad \varphi \in \mathbb{R}
$$

which leads to another form of Rodrigues' formula, that is valid for time-like vectors

$$
\mathcal{R}_{h}(\hat{\mathbf{n}}, \varphi)=\cos \varphi \mathcal{I}-(1-\cos \varphi) \hat{\mathbf{n}} \otimes(\eta \hat{\mathbf{n}})^{t}+\sin \varphi \hat{\mathbf{n}}^{\curlywedge}
$$


The so-called null or isotropic case $\zeta^{2}=0$ is discussed separately in Section 4 . Note that the vector parameter $\mathbf{c}$ can be projected using either $\operatorname{SU}(1,1)$ or $\operatorname{SL}(2, \mathbb{R})$ quaternion construction. In both cases we divide by $\zeta_{0}=\frac{1}{2} \operatorname{Tr} \zeta$ and the components are given respectively by

$$
\mathbf{c}=\frac{1}{\operatorname{Tr} \zeta}\left(\begin{array}{c}
\mathfrak{z}_{12}+\mathfrak{z}_{21} \\
\mathfrak{i}_{21}-\mathfrak{i}_{12} \\
\mathfrak{z}_{22}-\mathfrak{i}_{11}
\end{array}\right)_{\zeta \in \operatorname{SU}(1,1)} \quad \mathbf{c}=\frac{1}{\operatorname{Tr} \zeta}\left(\begin{array}{l}
\mathfrak{z}_{12}+\mathfrak{z}_{21} \\
\mathfrak{z}_{11}-\mathfrak{z}_{22} \\
\mathfrak{z}_{12}-\mathfrak{z}_{21}
\end{array}\right)_{\zeta \in \mathrm{SL}(2, \mathbb{R})}
$$

where $\mathfrak{z}_{i j}$ stand for the components of the quaternion $\zeta$, written as a $2 \times 2$ matrix. On the other hand, we may easily determine $\zeta$ from those of $\mathbf{c}$, using the fact that $\zeta$ is unit, which means $\zeta_{0}^{2}-\zeta^{2}=1$ and thus

$$
\mathbf{c}^{2}=\frac{\zeta_{0}^{2}-1}{\zeta_{0}^{2}} \Longrightarrow \zeta_{0}^{1,2}= \pm\left(1-\mathbf{c}^{2}\right)^{-\frac{1}{2}}, \quad \zeta^{1,2}=\zeta_{0}^{1,2} \mathbf{c}
$$

where the two signs correspond to the two sheets of the cover.

\subsection{Composition Law for the Vector Parameters}

Due to the multiplication table of the matrices in (1)

$$
\begin{gathered}
\mathbf{e}_{1} \mathbf{e}_{2}=-\mathbf{e}_{3}, \quad \mathbf{e}_{2} \mathbf{e}_{3}=\mathbf{e}_{1}, \quad \mathbf{e}_{3} \mathbf{e}_{1}=\mathbf{e}_{2}, \quad \mathbf{e}_{1}^{2}=\mathbf{e}_{2}^{2}=-\mathbf{e}_{3}^{2}=\mathbf{e}^{0} \\
\mathbf{e}_{i} \mathbf{e}_{j}+\mathbf{e}_{j} \mathbf{e}_{i}=0, \quad i, j=1,2,3
\end{gathered}
$$

we have a rather simple composition law for two pseudo-quaternions $\xi=\left(\xi_{0}, \boldsymbol{\xi}\right)$ and $\zeta=\left(\zeta_{0}, \zeta\right)$, namely

$$
\zeta \xi=\left(\zeta_{0} \xi_{0}+\boldsymbol{\zeta} \cdot \boldsymbol{\xi}, \zeta_{0} \boldsymbol{\xi}+\xi_{0} \boldsymbol{\zeta}+\boldsymbol{\zeta} \curlywedge \boldsymbol{\xi}\right) .
$$

In the spirit of projective geometry, we may think of $\xi_{1}, \xi_{2}$ and $\xi_{3}$ as the homogeneous coordinates of the $\mathbb{R P}^{3}$ - points $\mathbf{c}_{1}, \mathbf{c}_{2}$ and $\mathbf{c}_{3}$ respectively. In other words we have $^{2}\left(\stackrel{\circ}{\xi}_{i}, \boldsymbol{\xi}_{i}\right) \sim\left(1, \mathbf{c}_{i}\right)$ or $\mathbf{c}_{i}=\frac{\boldsymbol{\xi}_{i}}{\dot{\xi}_{i}}$, so the composition of two vector parameters may be obtained by projection of the composition in $\mathrm{SU}(1,1)$, i.e., the ratio

\footnotetext{
${ }^{2}$ For simplicity of notations we use $\dot{\xi}_{i}$ instead of $\left(\xi_{i}\right)_{0}$ for the scalar component of $\xi_{i}$
} 
between its vector and scalar part

$$
\left\langle\mathbf{c}_{2}, \mathbf{c}_{1}\right\rangle=\frac{\mathbf{c}_{2}+\mathbf{c}_{1}+\mathbf{c}_{2} \curlywedge \mathbf{c}_{1}}{1+\mathbf{c}_{2} \cdot \mathbf{c}_{1}}
$$

Then we naturally have

$$
\mathcal{R}_{h}\left(\mathbf{c}_{2}\right) \mathcal{R}_{h}\left(\mathbf{c}_{1}\right)=\mathcal{R}_{h}\left(\left\langle\mathbf{c}_{2}, \mathbf{c}_{1}\right\rangle\right), \quad \mathcal{R}_{h}(-\mathbf{c})=\mathcal{R}_{h}(\mathbf{c})^{-1}, \quad \mathcal{R}_{h}(0)=\mathcal{I}
$$

as in the Euclidean case. From the above it becomes clear why we arrange the indices in the backwards direction - this is because operator composition takes place from right to left.

If we want to decompose into three pseudo-rotations, first we need to notice that the scalar part of the product $\xi_{3} \xi_{2} \xi_{1}$ is given by

$$
\left(\xi_{3} \xi_{2} \xi_{1}\right)_{0}=\stackrel{\circ}{\xi}_{3} \stackrel{\circ}{\xi}_{2} \stackrel{\circ}{1}_{1}+\stackrel{\circ}{\xi}_{3} \boldsymbol{\xi}_{2} \cdot \boldsymbol{\xi}_{1}+\stackrel{\circ}{\xi}_{2} \boldsymbol{\xi}_{3} \cdot \boldsymbol{\xi}_{1}+\stackrel{\circ}{\xi}_{1} \boldsymbol{\xi}_{3} \cdot \boldsymbol{\xi}_{2}+\left(\boldsymbol{\xi}_{3}, \boldsymbol{\xi}_{2}, \boldsymbol{\xi}_{1}\right)
$$

where

$$
\left(\boldsymbol{\xi}_{3}, \boldsymbol{\xi}_{2}, \boldsymbol{\xi}_{1}\right)=\boldsymbol{\xi}_{3} \curlywedge \boldsymbol{\xi}_{2} \cdot \boldsymbol{\xi}_{1}
$$

denotes the triple product, i.e., the volume, spanned by $\boldsymbol{\xi}_{3}, \boldsymbol{\xi}_{2}$ and $\boldsymbol{\xi}_{1}$. As for the vector part, we have respectively

$$
\begin{aligned}
\operatorname{vec}\left(\xi_{3} \xi_{2} \xi_{1}\right)=\stackrel{\circ}{\xi}_{3} & \stackrel{\circ}{\xi}_{2} \boldsymbol{\xi}_{1}+\stackrel{\circ}{\xi}_{3} \stackrel{\circ}{\xi}_{1} \boldsymbol{\xi}_{2}+\stackrel{\circ}{\xi}_{2} \stackrel{\circ}{\xi}_{1} \boldsymbol{\xi}_{3}+\left(\boldsymbol{\xi}_{3} \cdot \boldsymbol{\xi}_{2}\right) \boldsymbol{\xi}_{1} \\
& +\stackrel{\circ}{\xi}_{3} \boldsymbol{\xi}_{2} \curlywedge \boldsymbol{\xi}_{1}+\stackrel{\circ}{\xi}_{2} \boldsymbol{\xi}_{3} \curlywedge \boldsymbol{\xi}_{1}+\stackrel{\circ}{\xi}_{1} \boldsymbol{\xi}_{3} \curlywedge \boldsymbol{\xi}_{2}+\left(\boldsymbol{\xi}_{3} \curlywedge \boldsymbol{\xi}_{2}\right) \curlywedge \boldsymbol{\xi}_{1}
\end{aligned}
$$

which can be projected to the composition $\mathbf{c}=\left\langle\mathbf{c}_{3}, \mathbf{c}_{2}, \mathbf{c}_{1}\right\rangle$ given as $\mathbf{c}=\frac{\mathbf{c}_{3}+\mathbf{c}_{2}+\mathbf{c}_{1}+\left(\mathbf{c}_{3} \cdot \mathbf{c}_{2}\right) \mathbf{c}_{1}+\mathbf{c}_{3} \curlywedge \mathbf{c}_{2}+\mathbf{c}_{3} \curlywedge \mathbf{c}_{1}+\mathbf{c}_{2} \curlywedge \mathbf{c}_{1}+\left(\mathbf{c}_{3} \curlywedge \mathbf{c}_{2}\right) \curlywedge \mathbf{c}_{1}}{1+\mathbf{c}_{3} \cdot \mathbf{c}_{2}+\mathbf{c}_{3} \cdot \mathbf{c}_{1}+\mathbf{c}_{2} \cdot \mathbf{c}_{1}+\left(\mathbf{c}_{3}, \mathbf{c}_{2}, \mathbf{c}_{1}\right)}$

Note that by construction we have for the double cross product signs, opposite to those in the Euclidean case

$$
\begin{aligned}
& \left(\mathbf{c}_{3} \curlywedge \mathbf{c}_{2}\right) \curlywedge \mathbf{c}_{1}=\left(\mathbf{c}_{1} \cdot \mathbf{c}_{2}\right) \mathbf{c}_{3}-\left(\mathbf{c}_{1} \cdot \mathbf{c}_{3}\right) \mathbf{c}_{2} \\
& \mathbf{c}_{3} \curlywedge\left(\mathbf{c}_{2} \curlywedge \mathbf{c}_{1}\right)=\left(\mathbf{c}_{2} \cdot \mathbf{c}_{3}\right) \mathbf{c}_{1}-\left(\mathbf{c}_{1} \cdot \mathbf{c}_{3}\right) \mathbf{c}_{2} .
\end{aligned}
$$

The sign difference is explained by the fact that in the flat Minkowski space we have a non-trivial metric $\eta$ to switch from upper to lower indices and vice versa. For example, the cross product of two vectors $c_{k}=(\mathbf{a} \times \mathbf{b})_{k}=\varepsilon_{j k l} a^{j} b^{l}$ is defined exactly as in the Euclidean case for the covariant (lower) indices, but the components of the expansion in the corresponding basis are $c^{i}=\eta^{i j} c_{j}$. Therefore, we use special notations for these operations to make the differences more apparent. 


\section{Generalized Euler Decomposition}

Here we utilize the expressions derived in the previous section for obtaining the vector decomposition of a generic pseudo-orthogonal transformation into two or three transformations of the same kind. We focus mostly on the Minkowski space model due to the close analogy with the Euclidean case. The results for $\operatorname{SU}(1,1)$ and $\operatorname{SL}(2, \mathbb{R})$ may be written using directly the explicit correspondence between pseudo-quaternions and vector parameters built in the previous section.

\subsection{The Case of Three Axes}

This is an analogue of the classical Euler decomposition for arbitrary axes in flat hyperbolic space

$$
\mathcal{R}_{h}(\tau \hat{\mathbf{n}})=\mathcal{R}_{h}\left(\tau_{3} \hat{\mathbf{c}}_{3}\right) \mathcal{R}_{h}\left(\tau_{2} \hat{\mathbf{c}}_{2}\right) \mathcal{R}_{h}\left(\tau_{1} \hat{\mathbf{c}}_{1}\right)
$$

where $\mathbf{c}=\tau \hat{\mathbf{n}}$ and $\mathbf{c}_{k}=\tau_{k} \hat{\mathbf{c}}_{k}$ are the vector parameters of the pseudo-rotations in the decomposition and $\hat{\mathbf{n}}, \hat{\mathbf{c}}_{k}$ - the corresponding quasi-unit vectors along the oriented axes. What we mean here by quasi-unit i s the following: since the hyperbolic flat metric in Minkowski space allows non-zero vectors to have positive, negative and vanishing scalar square, it is not always possible to normalize to unit "length". Instead, we have $\epsilon=\hat{\mathbf{n}} \cdot \hat{\mathbf{n}}= \pm 1$ in the space-like, respectively time-like case and $\epsilon=0$ in the isotropic one. Similarly, we use the notation $\epsilon_{k}=\hat{\mathbf{c}}_{k} \cdot \hat{\mathbf{c}}_{k}$.

In order to normalize an isotropic vector, we resort to a trick, known in Physics as Wick rotation - that is basically taking Euclidean rather than hyperbolic scalar product, or equivalently, multiplying the third component of the vector by a factor of $\sqrt{-1}$ (i.e., if $\epsilon=0$, we write $\mathbf{c}=\tau \hat{\mathbf{n}}$, where $\hat{\mathbf{n}}$ is unit in the Euclidean metric $\left.(\hat{\mathbf{n}}, \hat{\mathbf{n}})=\hat{n}_{1}^{2}+\hat{n}_{2}^{2}+\hat{n}_{3}^{2}=1\right)$.

Note that Euler's invariant axis theorem holds in the hyperbolic case as well (we comment on this fact into more detail in the next section) and from (13) it can be derived that $\hat{\mathbf{c}}_{1}$ and $\hat{\mathbf{c}}_{3}$ are eigenvectors of $\mathcal{R}_{h}\left(\tau_{1} \hat{\mathbf{c}}_{1}\right)$ and $\mathcal{R}_{h}\left(\tau_{3} \hat{\mathbf{c}}_{3}\right)$ respectively, with eigenvalue, equal to one. Thus we have

$$
\hat{\mathbf{c}}_{3} \cdot \mathcal{R}_{h}(\tau \hat{\mathbf{n}}) \hat{\mathbf{c}}_{1}=\hat{\mathbf{c}}_{3} \cdot \mathcal{R}_{h}\left(\tau_{2} \hat{\mathbf{c}}_{2}\right) \hat{\mathbf{c}}_{1}
$$

and if we introduce the notations

$$
\sigma_{i j}=\hat{\mathbf{c}}_{i} \cdot \mathcal{R}_{h}(\tau \hat{\mathbf{n}}) \hat{\mathbf{c}}_{j}, \quad \kappa_{i j}=\hat{\mathbf{c}}_{i} \cdot \hat{\mathbf{c}}_{j}, \quad \omega=\hat{\mathbf{c}}_{1} \cdot \hat{\mathbf{c}}_{2} \curlywedge \hat{\mathbf{c}}_{3}
$$


and take into account (15), the above leads to a quadratic equation for the unknown middle scalar parameter $\tau_{2}$

$$
\left[\epsilon_{2}\left(\sigma_{31}+\kappa_{31}\right)-2 \kappa_{12} \kappa_{23}\right] \tau_{2}^{2}-2 \omega \tau_{2}+\kappa_{31}-\sigma_{31}=0
$$

which has real roots whenever

$$
\Delta=\omega^{2}+\left[\epsilon_{2}\left(\sigma_{31}+\kappa_{31}\right)-2 \kappa_{12} \kappa_{23}\right]\left(\sigma_{31}-\kappa_{31}\right) \geq 0
$$

These solution, explicitly given by

$$
\tau_{2}^{ \pm}=\frac{\omega \pm \sqrt{\Delta}}{\epsilon_{2}\left(\sigma_{31}+\kappa_{31}\right)-2 \kappa_{12} \kappa_{23}}
$$

will be used as parameters in the equations, determining $\tau_{1}$ and $\tau_{3}$.

For the derivation of the latter, one may consider for example $\hat{\mathbf{c}}_{1} \cdot \mathcal{R}_{h}(\tau \hat{\mathbf{n}}) \hat{\mathbf{c}}_{1}$ and $\hat{\mathbf{c}}_{3} \cdot \mathcal{R}_{h}(\tau \hat{\mathbf{n}}) \hat{\mathbf{c}}_{3}$ in order to obtain the corresponding quadratic equations for the unknown scalar parameters with the help of (15).

However, this approach has the inconvenience of generating pseudo-solutions, that further need to be excluded, so we prefer to use another algorithm, which is linear. It is based on the vector parameter composition law, derived in the previous section. From (24) we may write

$$
\mathbf{c}_{1}=\left\langle-\mathbf{c}_{2},-\mathbf{c}_{3}, \mathbf{c}\right\rangle, \quad \mathbf{c}_{2}=\left\langle-\mathbf{c}_{3}, \mathbf{c},-\mathbf{c}_{1}\right\rangle, \quad \mathbf{c}_{3}=\left\langle\mathbf{c},-\mathbf{c}_{1},-\mathbf{c}_{2}\right\rangle
$$

and consider two successive projections - first we premultiply the $k^{\text {th }}$ equality above with $\hat{\mathbf{c}}_{k}^{\curlywedge}$ and then take (hyperbolic) dot product with $\hat{\mathbf{n}}$. Note that since $\hat{\mathbf{n}}$ is not allowed to be collinear with any of the $\hat{\mathbf{c}}_{k}$ 's (otherwise the decomposition would be trivial), the result is well defined and this trick allows for isolating one of the unknowns, thus obtaining explicit expressions for each pair of parameters as functions of the remaining one and the parameter of the compound pseudorotation. In particular we can express $\tau_{1}$ and $\tau_{3}$ in terms of $\tau$ and $\tau_{2}^{ \pm}$as

$$
\tau_{1}^{ \pm}=\frac{\left(\epsilon \kappa_{23}-\rho_{2} \rho_{3}\right) \tau \tau_{2}^{ \pm}+\tilde{\rho}^{1} \tau_{2}^{ \pm}}{\left(\rho_{1} \tilde{\rho}^{1}+\rho_{2} \tilde{\rho}^{2}\right) \tau \tau_{2}^{ \pm}+\left(\kappa_{23} \rho_{1}-\kappa_{13} \rho_{2}\right) \tau_{2}^{ \pm}+\left(\rho_{1} \rho_{3}-\epsilon \kappa_{13}\right) \tau+\tilde{\rho}^{2}}
$$

and

$$
\tau_{3}^{ \pm}=\frac{\left(\epsilon \kappa_{12}-\rho_{1} \rho_{2}\right) \tau \tau_{2}^{ \pm}+\tilde{\rho}^{3} \tau_{2}^{ \pm}}{\left(\rho_{2} \tilde{\rho}^{2}+\rho_{3} \tilde{\rho}^{3}\right) \tau \tau_{2}^{ \pm}+\left(\kappa_{12} \rho_{3}-\kappa_{13} \rho_{2}\right) \tau_{2}^{ \pm}+\left(\rho_{1} \rho_{3}-\epsilon \kappa_{13}\right) \tau+\tilde{\rho}^{2}}
$$


where we have used the notations

$$
\rho_{k}=\hat{\mathbf{c}}_{k} \cdot \hat{\mathbf{n}}, \quad \varepsilon_{i j k} \tilde{\rho}^{k}=\left(\hat{\mathbf{c}}_{i}, \hat{\mathbf{c}}_{j}, \hat{\mathbf{n}}\right)
$$

or to be more explicit

$$
\tilde{\rho}^{1}=\left(\hat{\mathbf{c}}_{2}, \hat{\mathbf{c}}_{3}, \hat{\mathbf{n}}\right), \quad \tilde{\rho}^{2}=\left(\hat{\mathbf{c}}_{3}, \hat{\mathbf{c}}_{1}, \hat{\mathbf{n}}\right), \quad \tilde{\rho}^{3}=\left(\hat{\mathbf{c}}_{1}, \hat{\mathbf{e}}_{2}, \hat{\mathbf{n}}\right) .
$$

We consider as an example the standard Euler decomposition (successive rotations about $O Z, O X$ and $O Z$ ) of a $\mathrm{SO}^{+}(2,1)$ matrix in the form

$$
\begin{gathered}
\left(\begin{array}{ccc}
\mathrm{c} \psi_{3} & -\mathrm{s} \psi_{3} & 0 \\
\mathrm{~s} \psi_{3} & \mathrm{c} \psi_{3} & 0 \\
0 & 0 & 1
\end{array}\right)\left(\begin{array}{ccc}
1 & 0 & 0 \\
0 & \mathrm{ch} \psi_{2} & -\mathrm{sh} \psi_{2} \\
0 & -\mathrm{sh} \psi_{2} & \mathrm{ch} \psi_{2}
\end{array}\right)\left(\begin{array}{ccc}
\mathrm{c} \psi_{1} & -\mathrm{s} \psi_{1} & 0 \\
\mathrm{~s} \psi_{1} & \mathrm{c} \psi_{1} & 0 \\
0 & 0 & 1
\end{array}\right) \\
=\left(\begin{array}{ccc}
\mathrm{c} \psi_{3} \mathrm{c} \psi_{1}-\mathrm{s} \psi_{3} \operatorname{ch} \psi_{2} \mathrm{~s} \psi_{1} & -\mathrm{c} \psi_{3} \mathrm{~s} \psi_{1}-\mathrm{s} \psi_{3} \operatorname{ch} \psi_{2} \mathrm{c} \psi_{1} & \mathrm{~s} \psi_{3} \operatorname{sh} \psi_{2} \\
\mathrm{~s} \psi_{3} \mathrm{c} \psi_{1}+\mathrm{c} \psi_{3} \operatorname{ch} \psi_{2} \mathrm{~s} \psi_{1} & \mathrm{c} \psi_{3} \operatorname{ch} \psi_{2} \mathrm{c} \psi_{1}-\mathrm{s} \psi_{3} \mathrm{~s} \psi_{1} & -\mathrm{c} \psi_{3} \operatorname{sh} \psi_{2} \\
-\operatorname{sh} \psi_{2} \mathrm{~s} \psi_{1} & -\operatorname{sh} \psi_{2} \mathrm{c} \psi_{1} & \operatorname{ch} \psi_{2}
\end{array}\right) .
\end{gathered}
$$

In front of the Greek letters we use here the abbreviations $\mathrm{s}=\sin , \mathrm{c}=\cos$, $\operatorname{sh}=\sinh$ and $\mathrm{ch}=$ cosh.Using the notations

$$
\tau_{2}=\tanh \frac{\psi_{2}}{2}, \quad \tau_{1,3}=\tan \frac{\psi_{1,3}}{2}
$$

we easily obtain the (projective) compound vector parameter in the form

$$
\mathbf{c}=\frac{1}{1-\tau_{1} \tau_{3}}\left(\begin{array}{c}
\tau_{2}\left(1+\tau_{1} \tau_{3}\right) \\
\tau_{2}\left(\tau_{3}-\tau_{1}\right) \\
\tau_{1}+\tau_{3}
\end{array}\right)
$$

and hence by the sign of the expression

$$
\mathbf{c}^{2} \sim \tau_{2}^{2}\left(1+\tau_{1}^{2}\right)\left(1+\tau_{3}^{2}\right)-\left(\tau_{1}+\tau_{3}\right)^{2}
$$

determine whether it is time-like, space-like or null (respectively whether the transformation thus obtained is elliptic, hyperbolic or parabolic).

We may also decompose using (28), (30) and (31). Note that in this particular case we have $\kappa_{12}=\kappa_{23}=0, \kappa_{13}=-1, \rho_{1}=\rho_{3}=\hat{n}_{3}=-\hat{n}^{3}, \rho_{2}=\hat{n}_{1}$, $\tilde{\rho}^{3}=-\tilde{\rho}^{1}=\hat{n}^{2}, \tilde{\rho}^{2}=0, \epsilon_{1}=\epsilon_{3}=-1, \epsilon_{2}=1$ and $\sigma_{31}=\mathcal{R}_{33}=-\mathcal{R}_{3}^{3}$, where $\mathcal{R}_{i j}$ and $\mathcal{R}_{j}^{i}$ are the components of $\mathcal{R}_{h}$ in the standard basis and the sign change is due to the fact that we use the metric $\eta$ to switch from lower to upper indices and vice versa. The above explicit form of the matrix gives the $\mathcal{R}_{j}^{i}$, s. With all this in 
mind, we easily obtain

$$
\tau_{2}^{ \pm}= \pm \sqrt{\frac{\mathcal{R}_{3}^{3}-1}{\mathcal{R}_{3}^{3}+1}}
$$

which gives $\tau_{2}^{ \pm}= \pm \tanh \frac{\psi_{2}}{2}$ using elementary hyperbolic trigonometry and the fact that $\mathcal{R}_{3}^{3}=\operatorname{ch} \psi_{2}$. Furthermore we have

$\tau_{1}^{ \pm}=\frac{\left(\hat{n}^{1} \hat{n}^{3} \tau-\hat{n}^{2}\right) \tau_{2}^{ \pm}}{\left(\hat{n}^{1}+\hat{n}^{2} \hat{n}^{3} \tau\right) \tau_{2}^{ \pm}+\left(\epsilon+\left(\hat{n}^{3}\right)^{2}\right) \tau}, \quad \tau_{3}^{ \pm}=\frac{\left(\hat{n}^{1} \hat{n}^{3} \tau+\hat{n}^{2}\right) \tau_{2}^{ \pm}}{\left(\hat{n}^{1}-\hat{n}^{2} \hat{n}^{3} \tau\right) \tau_{2}^{ \pm}+\left(\epsilon+\left(\hat{n}^{3}\right)^{2}\right) \tau}$

Alternatively, we may consider the classical Bryan decomposition about the axes $O X, O Y$ and $O Z$ which can be written explicitly as

$$
\begin{gathered}
\left(\begin{array}{ccc}
\mathrm{c} \psi_{3} & -\mathrm{s} \psi_{3} & 0 \\
\mathrm{~s} \psi_{3} & \mathrm{c} \psi_{3} & 0 \\
0 & 0 & 1
\end{array}\right)\left(\begin{array}{ccc}
\mathrm{ch} \psi_{2} & 0 & \mathrm{sh} \psi_{2} \\
0 & 1 & 0 \\
\operatorname{sh} \psi_{2} & 0 & \mathrm{ch} \psi_{2}
\end{array}\right)\left(\begin{array}{ccc}
1 & 0 & 0 \\
0 & \mathrm{ch} \psi_{1} & -\operatorname{sh} \psi_{1} \\
0 & -\operatorname{sh} \psi_{1} & \operatorname{ch} \psi_{1}
\end{array}\right) \\
=\left(\begin{array}{ccc}
\mathrm{c} \psi_{3} \operatorname{ch} \psi_{2} & -\mathrm{s} \psi_{3} \operatorname{ch} \psi_{1}-\mathrm{c} \psi_{3} \operatorname{sh} \psi_{2} \operatorname{sh} \psi_{1} & \mathrm{~s} \psi_{3} \operatorname{sh} \psi_{1}+\mathrm{c} \psi_{3} \operatorname{sh} \psi_{2} \operatorname{ch} \psi_{1} \\
\mathrm{~s} \psi_{3} \operatorname{ch} \psi_{2} & \mathrm{c} \psi_{3} \operatorname{ch} \psi_{1}-\operatorname{s} \psi_{3} \operatorname{sh} \psi_{2} \operatorname{sh} \psi_{1} & \mathrm{~s} \psi_{3} \operatorname{sh} \psi_{2} \operatorname{ch} \psi_{1}-\operatorname{c} \psi_{3} \operatorname{sh} \psi_{1} \\
\operatorname{sh} \psi_{2} & -\operatorname{ch} \psi_{2} \operatorname{sh} \psi_{1} & \operatorname{ch} \psi_{2} \operatorname{ch} \psi_{1}
\end{array}\right)
\end{gathered}
$$

and for the vector parameter we have

$$
\mathbf{c}=\frac{1}{1-\tau_{1} \tau_{2} \tau_{3}}\left(\begin{array}{c}
\tau_{1}-\tau_{2} \tau_{3} \\
\tau_{2}+\tau_{1} \tau_{3} \\
\tau_{3}+\tau_{1} \tau_{2}
\end{array}\right)
$$

If we denote $\boldsymbol{\tau}=\left(\tau_{1}, \tau_{2}, \tau_{3}\right)^{t}$ and $\tilde{\boldsymbol{\tau}}=\left(-\tau_{2} \tau_{3}, \tau_{1} \tau_{3}, \tau_{1} \tau_{2}\right)^{t}$, the above is proportional to the vector sum $\tau+\tilde{\tau}$, so in order to determine the type of the composite vector (resp. transformation) we need the sign of the expression

$$
\mathbf{c}^{2} \sim(\boldsymbol{\tau}+\tilde{\boldsymbol{\tau}})^{2}=\boldsymbol{\tau}^{2}+\tilde{\boldsymbol{\tau}}^{2}-2 \tau_{1} \tau_{2} \tau_{3}
$$

In this example we have $\kappa=\eta, \omega=1, \rho_{k}=\hat{n}_{k}, \tilde{\rho}^{k}=\hat{n}^{k}, \epsilon_{1}=\epsilon_{2}=1, \epsilon_{3}=-1$ and $\sigma_{31}=\mathcal{R}_{31}=-\mathcal{R}_{1}^{3}=-\operatorname{sh} \psi_{2}$, so we easily obtain

$$
\tau_{2}^{ \pm}=-\frac{1 \pm \sqrt{1+\left(\mathcal{R}_{1}^{3}\right)^{2}}}{\mathcal{R}_{1}^{3}}=-\frac{1 \pm \operatorname{ch} \psi_{2}}{\operatorname{sh} \psi_{2}}=\left\{-\operatorname{coth} \frac{\psi_{2}}{2}, \tanh \frac{\psi_{2}}{2}\right\}
$$


and for the other two scalar parameters

$\tau_{1}^{ \pm}=\frac{\left(\hat{n}^{1}+\hat{n}^{2} \hat{n}^{3} \tau\right) \tau_{2}^{ \pm}}{\left(\epsilon+\left(\hat{n}^{3}\right)^{2}\right) \tau \tau_{2}^{ \pm}-\hat{n}^{1} \hat{n}^{3} \tau+\hat{n}^{2}}, \quad \tau_{3}^{ \pm}=\frac{\left(\hat{n}^{3}-\hat{n}^{1} \hat{n}^{2} \tau\right) \tau_{2}^{ \pm}}{\left(\epsilon-\left(\hat{n}^{1}\right)^{2}\right) \tau \tau_{2}^{ \pm}-\hat{n}^{1} \hat{n}^{3} \tau+\hat{n}^{2}}$

which can easily be rewritten for the covariant components $\mathcal{R}_{i j}$ and $\hat{n}_{j}$, as well as in terms of trigonometric functions of the $\psi_{i}$ 's.

For a more detailed derivation of the analogous expressions in the Euclidean case we refer to [5].

To sum up, as it has been shown in [18], one may always decompose into three transformations with respect to non-collinear axes (actually, we demand only the second axis not to be collinear with the other two) among which one needs to be space-like in the generic case. Apart from that, these axes may be arbitrary, as long as the positive discriminant condition (27) is fulfilled.

\subsection{The Case of Two Axes}

We are also interested in deriving explicit formulae for a decomposition of the type

$$
\mathcal{R}_{h}(\tau \hat{\mathbf{n}})=\mathcal{R}_{h}\left(\tau_{2} \hat{\mathbf{c}}_{2}\right) \mathcal{R}_{h}\left(\tau_{1} \hat{\mathbf{c}}_{1}\right)
$$

together with the necessary and sufficient conditions for its existence.

Following the same idea as before, we easily obtain

$$
\sigma_{21}=\hat{\mathbf{c}}_{2} \cdot \mathcal{R}_{h}(\tau \hat{\mathbf{n}}) \hat{\mathbf{c}}_{1}=\hat{\mathbf{c}}_{2} \cdot \hat{\mathbf{c}}_{1}=\kappa_{21}
$$

which plays the role of such condition.

As for the expressions for $\tau_{1}$ and $\tau_{2}$ we use the explicit form (22) of

$$
\mathbf{c}=\left\langle\mathbf{c}_{2}, \mathbf{c}_{1}\right\rangle
$$

which we multiply on the left by $\hat{\mathbf{n}}^{\curlywedge}$ and then consider hyperbolic scalar products with $\hat{\mathbf{c}}_{1}$ and $\hat{\mathbf{c}}_{1}$ respectively to obtain (assuming $\tau_{1,2} \neq 0$ )

$$
\tau_{1}=\frac{\tilde{\rho}^{3}}{\epsilon_{1} \rho_{2}-\kappa_{12} \rho_{1}}, \quad \tau_{2}=\frac{\tilde{\rho}^{3}}{\epsilon_{2} \rho_{1}-\kappa_{12} \rho_{2}} .
$$

In the Euclidean case of pure $\mathrm{SO}(3)$ rotations we have similar expressions with $\epsilon_{1}=\epsilon_{2}=1$ and $\tilde{\rho}_{3}=-\tilde{\rho}^{3}$.

$$
\tau_{1}=\frac{\tilde{\rho}_{3}}{\kappa_{12} \rho_{1}-\rho_{2}}, \quad \tau_{2}=\frac{\tilde{\rho}_{3}}{\kappa_{12} \rho_{2}-\rho_{1}}
$$


and they ought to be considered instead of formula (29) in [5], which is mistaken for some reason (in the notations of [5] we have $u, v$ instead of $\tau_{1}, \tau_{2}$ ).

\subsection{Degenerate Solutions}

There is one particular situation in which one cannot determine (30) and (31) independently, but only a certain combination of these two, due to a lack of sufficient information. This is an analogue of the classical gimbal lock - a phenomenon, well known in the applications.

The condition for such a degenerate type of solution is rather simple

$$
\hat{\mathbf{c}}_{3}= \pm \mathcal{R}_{h}(\mathbf{c}) \hat{\mathbf{c}}_{1}
$$

and in that case (24) takes the form

$$
\mathcal{R}_{h}(\mathbf{c})=\mathcal{R}_{h}\left( \pm \tau_{3} \mathcal{R}_{h}(\mathbf{c}) \hat{\mathbf{c}}_{1}\right) \mathcal{R}_{h}\left(\tau_{2} \hat{\mathbf{c}}_{2}\right) \mathcal{R}_{h}\left(\tau_{1} \hat{\mathbf{c}}_{1}\right)
$$

which can also be written as

$$
\mathcal{R}_{h}\left(\tau_{2} \hat{\mathbf{c}}_{2}\right) \mathcal{R}_{h}\left(\tau_{1} \hat{\mathbf{c}}_{1}\right)=\mathcal{R}_{h}\left(\mp \tau_{3} \mathcal{R}_{h}(\mathbf{c}) \hat{\mathbf{c}}_{1}\right) \mathcal{R}_{h}(\mathbf{c})=\mathcal{R}_{h}(\mathbf{c}) \mathcal{R}_{h}\left(\mp \tau_{3} \hat{\mathbf{c}}_{1}\right)
$$

where for the last equality we use the well-known relation

$$
\mathcal{R}(\mathbf{c}) \mathcal{R}(\mathbf{a}) \mathcal{R}(\mathbf{c})^{-1}=\mathcal{R}(\mathcal{R}(\mathbf{c}) \mathbf{a})
$$

that is equally valid for Euclidean and hyperbolic rotations.

Multiplying both sides of (39) on the right with $\mathcal{R}_{h}\left( \pm \tau_{3} \hat{\mathbf{c}}_{1}\right)$ we end up with

$$
\mathcal{R}_{h}(\mathbf{c})=\mathcal{R}_{h}\left(\tau_{2} \hat{\mathbf{c}}_{2}\right) \mathcal{R}_{h}\left(\left\langle\tau_{1} \hat{\mathbf{c}}_{1} \pm \tau_{3} \hat{\mathbf{c}}_{1}\right\rangle\right)=\mathcal{R}_{h}\left(\tau_{2} \hat{\mathbf{c}}_{2}\right) \mathcal{R}_{h}\left(\tau_{1}^{\prime} \hat{\mathbf{c}}_{1}\right)
$$

where

$$
\tau_{1}^{\prime}=\frac{\tau_{1} \pm \tau_{3}}{1 \pm \epsilon_{1} \tau_{1} \tau_{3}}
$$

according to (22).

We see that the problem is reduced to decomposition into a pair of rotations and since (41) guarantees that the corresponding condition (34) is fulfilled, we may use (35) to obtain

$$
\tau_{2}=\frac{\tilde{\rho}^{3}}{\epsilon_{2} \rho_{1}-\kappa_{12} \rho_{2}}, \quad \frac{\tau_{1} \pm \tau_{3}}{1 \pm \epsilon_{1} \tau_{1} \tau_{3}}=\frac{\tilde{\rho}^{3}}{\epsilon_{1} \rho_{2}-\kappa_{12} \rho_{1}} .
$$


The first equation above determines $\tau_{2}$ uniquely, but the second one fails to do so with the remaining parameters $\tau_{1}$ and $\tau_{3}$. Instead, it provides a single relation for them, which allows each to be expressed from the other, or in other words we end up with a one-parameter degenerate solution, just as in the Euclidean case (see also [27]).

\subsection{The Trivial Element}

Here we consider a decomposition of the trivial element in $\mathrm{SO}^{+}(2,1)$ (the identity transformation) which cannot be assigned a particular direction $\hat{\mathbf{n}}$, since the scalar (and respectively the vector) parameter is identically zero. Therefore neither $\boldsymbol{\rho}$ nor $\tilde{\boldsymbol{\rho}}$ is well defined and we cannot use the results, obtained above (formulae (28), (30) and (31)), so instead we take advantage of the fact that each of the pseudo-rotations in the decomposition can be expressed as a superposition of the remaining two

$$
\mathbf{c}_{1}=\left\langle-\mathbf{c}_{2},-\mathbf{c}_{3}\right\rangle, \quad \mathbf{c}_{2}=\left\langle-\mathbf{c}_{3},-\mathbf{c}_{1}\right\rangle, \quad \mathbf{c}_{3}=\left\langle-\mathbf{c}_{1},-\mathbf{c}_{2}\right\rangle
$$

since $\langle\mathbf{a}, 0\rangle=\langle 0, \mathbf{a}\rangle=\mathbf{a}$. Then we multiply the $k^{t h}$ equality in the system with $\hat{\mathbf{c}}_{k}^{\curlywedge}$ and consider (hyperbolic) dot products with $\hat{\mathbf{c}}_{2}$ for the first, respectively $\hat{\mathbf{c}}_{3}$ for the second and $\hat{\mathbf{c}}_{1}$ for the third relation to obtain

$$
\begin{aligned}
& \left(\kappa_{12} \kappa_{23}-\epsilon_{2} \kappa_{13}\right) \tau_{2} \tau_{3}+\omega \tau_{3}=0 \\
& \left(\kappa_{13} \kappa_{23}-\epsilon_{3} \kappa_{12}\right) \tau_{1} \tau_{3}+\omega \tau_{1}=0 \\
& \left(\kappa_{12} \kappa_{13}-\epsilon_{1} \kappa_{23}\right) \tau_{1} \tau_{2}+\omega \tau_{2}=0
\end{aligned}
$$

which always has the trivial solution $\tau=0$ and for $\omega \neq 0$ there is one more

$$
\tau_{1}=\frac{\omega}{\epsilon_{1} \kappa_{23}-\kappa_{12} \kappa_{13}}, \quad \tau_{2}=\frac{\omega}{\epsilon_{2} \kappa_{13}-\kappa_{12} \kappa_{23}}, \quad \tau_{3}=\frac{\omega}{\epsilon_{3} \kappa_{12}-\kappa_{13} \kappa_{23}} .
$$

In the case $\omega=0$, just as in its Euclidean analogue (see [5]), there is a nontrivial solution if and only if $\hat{\mathbf{c}}_{1}= \pm \hat{\mathbf{c}}_{3}$. This solution, however, is not uniquely determined - we have $\tau_{2}^{+}=\tau_{2}^{-}=0$ and $\tau_{1}=\mp \tau_{3}$, so that the two non-trivial transformations in the decomposition are mutually inverse, which provides a good example for a degenerate solution.

\subsection{Divergencies}

We note that the denominator in (22) is very likely to vanish in certain cases and there is nothing disturbing about that. Since we are dealing with projective quantities, after all, division by zero is well justified operation. In order to avoid the 
inconvenience of dealing with vectors of infinite magnitude, however, one may simply lift up back to the universal cover. Diverging vector parameters correspond to pseudo-quaternions with vanishing scalar part $\zeta_{0}=0 \Rightarrow \zeta^{2}=-1$, so they may appear only if the compound vector is time-like and thus the transformation associated with it - elliptic. In particular, if the invariant axis is collinear with $O Z$, we encounter a half-turn (rotation by an angle $\pi$ ). More generally, we may have a product of a pure rotation and a Lorentz boost. Here we consider different configurations of compositions that produce such a transformation. The condition for this to happen, as can be seen from (22), has the simple form

$$
1+\mathbf{c}_{1} \cdot \mathbf{c}_{2}=0
$$

In the parabolic case for example, we may think of the vector parameters as generated by $\mathbb{R}^{2}$ that can be written as complex numbers

$$
\mathbb{C} \ni z_{k}=x_{k}+\mathrm{i} y_{k} \longrightarrow \mathbf{c}_{k}=\left(x_{k}, y_{k}, \sqrt{x_{k}^{2}+y_{k}^{2}}\right)=\left(z_{k},\left|z_{k}\right|\right), \quad k=1,2
$$

and then the hyperbolic dot product of the $\mathbf{c}_{k}$ 's is given by

$$
\mathbf{c}_{1} \cdot \mathbf{c}_{2}=\Re\left(z_{1} \bar{z}_{2}\right)-\left|z_{1}\right|\left|z_{2}\right|=\left|z_{1}\right|\left|z_{2}\right|(\cos \phi-1), \quad \phi=\arg z_{2}-\arg z_{1}
$$

so in order to have (47) satisfied we need for the angle between the two vectors to obey the constraint

$$
\cos \phi=1-\frac{1}{\left|z_{1}\right|\left|z_{2}\right|} .
$$

In particular, we may have $\left|z_{1}\right|=\left|z_{2}\right|=1$ and $\phi=\frac{\pi}{2}$, or let $\left|z_{1}\right|,\left|z_{2}\right| \rightarrow \infty$ which leads to $\phi \rightarrow 0$, once more leaving two out of three independent parameters. Another example would be to restrict to one-parameter submanifold by the following contruction

$\mathbf{c}_{1}=(s, s+1, \sqrt{2 s(s+1)+1}), \quad \mathbf{c}_{2}=(s+1, s, \sqrt{2 s(s+1)+1}), \quad s \in \mathbb{R}$.

In the elliptic and hyperbolic cases, we deal with co-dimension one submanifolds since the restriction to the vector norm is given by an inequality, rather than equality. Compositions of pure boosts that result in elliptic transformations are interesting for the applications (see Section 5) and as for the case of rotations, they have been thoroughly studied in [5]. There we make use of l'Hôpital's rule to derive the scalar parameters of the generalized Euler decomposition, when the compound transformation happens to be a half-turn. Similarly, we may study (30) and (31) in 
the limit $\tau \rightarrow \infty$ to obtain

$$
\begin{aligned}
\tau_{1}^{ \pm} & =\frac{\left(\epsilon \kappa_{23}-\rho_{2} \rho_{3}\right) \tau_{2}^{ \pm}}{\left(\rho_{1} \tilde{\rho}^{1}+\rho_{2} \tilde{\rho}^{2}\right) \tau_{2}^{ \pm}+\rho_{1} \rho_{3}-\epsilon \kappa_{13}} \\
\tau_{3}^{ \pm} & =\frac{\left(\epsilon \kappa_{12}-\rho_{1} \rho_{2}\right) \tau_{2}^{ \pm}}{\left(\rho_{2} \tilde{\rho}^{2}+\rho_{3} \tilde{\rho}^{3}\right) \tau_{2}^{ \pm}+\rho_{1} \rho_{3}-\epsilon \kappa_{13}}
\end{aligned}
$$

It could happen in particular that $\tau_{2} \rightarrow \infty$ as well (if $\sigma_{31} \neq \kappa_{31}$ and $\Delta=0 \neq \omega$ ). Then we apply l'Hôpital's rule once more and end up with

$$
\tau_{1}=\frac{\epsilon \kappa_{23}-\rho_{2} \rho_{3}}{\rho_{1} \tilde{\rho}^{1}+\rho_{2} \tilde{\rho}^{2}}, \quad \tau_{3}=\frac{\epsilon \kappa_{12}-\rho_{1} \rho_{2}}{\rho_{2} \tilde{\rho}^{2}+\rho_{3} \tilde{\rho}^{3}} .
$$

This simple tricks works also for compositions of the type (22) - if either $\mathbf{c}_{1}$ or $\mathbf{c}_{2}$ has divergent pseudo-norm (i.e., $\tau_{1} \rightarrow \infty$ or $\tau_{2} \rightarrow \infty$ ), then it is straightforward to write

$$
\left\langle\mathbf{c}_{2}, \mathbf{c}_{1}\right\rangle \rightarrow \frac{\mathbf{c}_{1}+\mathbf{c}_{2} \curlywedge \mathbf{c}_{1}}{\mathbf{c}_{2} \cdot \mathbf{c}_{1}}, \quad\left\langle\mathbf{c}_{2}, \mathbf{c}_{1}\right\rangle \rightarrow \frac{\mathbf{c}_{2}+\mathbf{c}_{2} \curlywedge \mathbf{c}_{1}}{\mathbf{c}_{2} \cdot \mathbf{c}_{1}}
$$

in the former and in the latter case respectively, so that the divergencies cancel out. On the other hand, if both scalar parameters tend to infinity simultaneously, then we simply have

$$
\left\langle\mathbf{c}_{2}, \mathbf{c}_{1}\right\rangle \rightarrow \frac{\mathbf{c}_{2} \curlywedge \mathbf{c}_{1}}{\mathbf{c}_{2} \cdot \mathbf{c}_{1}}
$$

similarly to the Euclidean case.

\section{Null Vectors and Invariant Axes}

In this section we discuss the hyperbolic analogue of Euler's fixed axis theorem and then focus on the behavior of parabolic and elliptic representatives of $\mathrm{SO}^{+}(2,1)$.

\subsection{Euler's Fixed Axis Theorem}

First we remind that in the Euclidean case (see e.g. [5, 26]) there is a theorem due to Euler, stating that a special orthogonal transformation in $\mathbb{R}^{2 k+1}$ has an invariant axis, determined by the eigenvector, corresponding to the eigenvalue one. The latter is unique except in the trivial case - the identity operator, for which the eigenspace of such vectors coincides with the imbedding space $\mathbb{R}^{2 k+1}$.

One possible proof uses the fact that skew-symmetric matrices in odd dimensions are singular and thus have a zero eigenvalue. Since they constitute the Lie algebra 
$\mathfrak{s o}(2 k+1)$ of the corresponding group of rotations, for the latter we have a unit eigenvalue given by the exponential map, which is either simple in the generic case, or triple in the trivial one.

Similarly, we may construct the standard matrix representation of $\mathfrak{s o}(2,1)$ from the adjoint action of the generators $\mathbf{e}_{k}$ (or $\tilde{\mathbf{e}}_{k}$ ), which allows for expressing a generic element $\mathcal{A} \in \mathfrak{s o}(2,1)$ as

$$
\mathcal{A}(\mathbf{x})=x^{k} \operatorname{ad}\left(\mathbf{e}_{k}\right)=\mathbf{x}^{\curlywedge}=\left(\begin{array}{ccc}
0 & -x_{3} & x_{2} \\
x_{3} & 0 & -x_{1} \\
x_{2} & -x_{1} & 0
\end{array}\right) .
$$

This gives for the eigenvalues $\lambda_{1}=0$ and $\lambda_{2,3}= \pm \sqrt{x_{1}^{2}+x_{2}^{2}-x_{3}^{2}}= \pm \sqrt{\mathbf{x} \cdot \mathbf{x}}$ and we may consider some specific cases:

1. For $x_{3}=0$ and $\mathbf{x} \in \mathbb{R}^{2}$, that is a particular example of a space-like vector (pointing outside the light cone), we have distinct real eigenvalues and eigenvectors. Moreover $\mathcal{A}$ is symmetric in this case, so it can easily be put into diagonal form.

2. When $x_{1}=x_{2}=0, x_{3} \neq 0$ - the vector is time-like (inside the light cone) and we end up with a skew-symmetric matrix that generates a rotation. The eigenvalues are 0 and $\pm \mathrm{i} x_{3}$ and the real eigenvector is oriented in the $z$ direction (we end up with a pure rotation about $O Z$ ).

3. Finally, if $\mathbf{x}$ is null $(\mathbf{x} \cdot \mathbf{x}=0)$, we end up with a triple eigenvalue $\lambda=0$ and a triple eigenvector $\mathrm{x}$ as already discussed.

The above present particular examples for generators of hyperbolic, elliptic and respectively parabolic transformations. More generally, similarly to $\operatorname{SL}(2, \mathbb{R})$, we have an easy criterion to distinguish between these cases, namely $\mathcal{R}_{h}$ is

1. hyperbolic if $\operatorname{Tr}\left(\mathcal{R}_{h}\right)>3$

2. elliptic if $\operatorname{Tr}\left(\mathcal{R}_{h}\right)<3$

3. parabolic if $\operatorname{Tr}\left(\mathcal{R}_{h}\right)=3$

which can be used to determine the type of its vector parameter: it is space-like in the first case, time-like in the second one and null or isotropic (also called lightlike) in the third case.

After we have determined the type of the vector-parameter, it is not difficult to determine its direction and magnitude in a way, similar to the one, proposed in [26] 
for the $\mathrm{SO}(3)$ case. Actually, as long as $\varphi \neq 0$ and in the pure rotational case also $\varphi \neq \pi$, we can always determine $\mathbf{c}$, at least up to a scale factor, from the term $\mathbf{c}^{\curlywedge}$ in (15). More precisely we have

$$
\hat{\mathbf{n}}=\frac{1-\epsilon \tau^{2}}{2 \tau}\left(\begin{array}{r}
-\mathcal{R}_{23} \\
\mathcal{R}_{13} \\
-\mathcal{R}_{12}
\end{array}\right)
$$

where $\mathcal{R}_{i j}$ denote the matrix entries of $\mathcal{R}_{h}(\tau \hat{\mathbf{n}})$ in the standard basis and the prefactor on the right is equal to $(\sinh \varphi)^{-1},(\sin \varphi)^{-1}$ or $\varphi^{-1}$ for $\epsilon= \pm 1$ and $\epsilon=0$ respectively. In the elliptic case this does not determine the angle completely, so we need also $\cos \varphi$ which is easy to find from $\operatorname{Tr}\left(\mathcal{R}_{h}\right)=2 \cos \varphi+1$. One specific example is the so-called half turn $\mathcal{O}(\hat{\mathbf{n}})=2 \hat{\mathbf{n}} \otimes \hat{\mathbf{n}}^{t}-\mathcal{I}$.

With this technique we can always determine the vector parameter (or the angle and axis) for a $\mathrm{SO}^{+}(2,1)$ transformation and vice versa.

\subsection{The Parabolic Case}

In Minkowski space each null vector lies in its orthogonal complement - something that has no analogue in Euclidean geometry. When we use such vector in (15), the exponential map gives for the corresponding group element $\mathcal{R}_{h}(\mathbf{x})=\exp \mathcal{A}(\mathbf{x})$ a triple eigenvalue $\mathrm{e}^{0}=1$ although there is a single invariant axis, determined by $\hat{\mathbf{x}}$. We note that for a generic $\mathbf{x} \in \mathbb{R}^{3}$ we have

$$
\mathcal{A}(\mathbf{x})=\mathbf{x}^{\curlywedge}, \quad \mathcal{A}^{2}(\mathbf{x})=(\mathbf{x} \cdot \mathbf{x}) \mathcal{I}-\mathbf{x} \otimes(\eta \mathbf{x})^{t}, \quad \mathcal{A}^{3}(\mathbf{x})=(\mathbf{x} \cdot \mathbf{x}) \mathcal{A}(\mathbf{x})
$$

which leads back to different versions of Rodrigues' formula, based on the matrix exponent $\mathcal{R}_{h}(\mathbf{x})=\exp \mathcal{A}(\mathbf{x})$ if we put $\mathbf{x}=\varphi \hat{\mathbf{n}}$. However, this works only if $\mathrm{x} \cdot \mathrm{x} \neq 0$ - for a null vector we end up with

$$
\mathcal{R}_{h}(\mathbf{x})=\exp \mathcal{A}(\mathbf{x})=\mathcal{I}+\mathbf{x}^{\curlywedge}-\frac{1}{2} \mathbf{x} \otimes(\eta \mathbf{x})^{t}, \quad \mathbf{x} \cdot \mathbf{x}=0
$$

since all higher order terms vanish.

According to our previous notations, however, we have $\mathbf{x}=\varphi \hat{\mathbf{n}}$ and thus

$$
\mathcal{R}_{h}(\varphi \hat{\mathbf{n}})=\exp \mathcal{A}(\varphi \hat{\mathbf{n}})=\mathcal{I}+\varphi \hat{\mathbf{n}}^{\curlywedge}-\frac{\varphi^{2}}{2} \hat{\mathbf{n}} \otimes(\eta \hat{\mathbf{n}})^{t}, \quad \hat{\mathbf{n}} \cdot \hat{\mathbf{n}}=0
$$


which can be used in an appropriate scalar product to obtain the analogue of (28) for the parabolic case

$$
\varphi^{ \pm}=\frac{-\omega \pm \sqrt{\Delta}}{\kappa_{12} \kappa_{23}}
$$

with

$$
\Delta=\omega^{2}+2\left(\kappa_{31}-\sigma_{31}\right) \kappa_{12} \kappa_{23} \geq 0
$$

being the necessary and sufficient condition for the existence of real solutions.

We note that in this case the Euler substitution is not justified, since we are at a separatrix between elliptic and hyperbolic regions. That is why the parametrizations with the "angle" $\varphi$ appears most natural - they leads to the Rodrigues' formula and we can write $\varphi$ instead of $\sin \varphi(\sinh \varphi), 1$ for $\cos \varphi($ respectively $\cosh \varphi)$ and corrected value $\frac{\varphi^{2}}{2}$ for $1-\cos \varphi$ (or $\left.\cosh \varphi-1\right)$.

From this point of view (54) is a substitute for (13) and (17) for the parabolic case. It also allows to use $\tau=\frac{\varphi}{2}$ so that (15) and (22) make sense as well.

On the other hand setting $\epsilon_{2}=0$, in (28) we easily derive the relation

$$
\tau_{2}^{ \pm}=\frac{-\omega \pm \sqrt{\Delta}}{2 \kappa_{12} \kappa_{23}}
$$

which is in complete accordance with the corresponding expressions obtained above. Now let us see how composition of parabolic elements works on the level of vector parameters. Applying (22) with $\mathbf{c}_{i}=\frac{\varphi_{i}}{2} \hat{\mathbf{c}}_{i},\left(\hat{\mathbf{c}}_{i}, \hat{\mathbf{c}}_{i}\right)=1$ we end up with

$$
\left\langle\mathbf{c}_{2}, \hat{\mathbf{c}}_{1}\right\rangle=\frac{1}{2}\left(\varphi_{1} \hat{\mathbf{c}}_{1}+\varphi_{2} \hat{\mathbf{c}}_{2}+\frac{\varphi_{1} \varphi_{2}}{2} \hat{\mathbf{c}}_{2} \curlywedge \hat{\mathbf{c}}_{1}\right)
$$

in the case $\hat{\mathbf{c}}_{1} \cdot \hat{\mathbf{c}}_{2}=0$. In particular for $\hat{\mathbf{c}}_{1}=\hat{\mathbf{c}}_{2}$ the above reduces to

$$
\varphi=\varphi_{1}+\varphi_{2} .
$$

On the other hand, one may consider the asymptotic behavior of the composition for small and large values of the parameters $\varphi_{i}$. In the first case the composition is reduced to a simple vector summation

$$
\mathbf{c} \sim \mathbf{c}_{1}+\mathbf{c}_{2}, \quad \varphi_{1}, \varphi_{2} \rightarrow 0
$$

while in the second one, we have a formula, familiar from the rotational case

$$
\mathbf{c} \sim \frac{\mathbf{c}_{2} \curlywedge \mathbf{c}_{1}}{\mathbf{c}_{2} \cdot \mathbf{c}_{1}}, \quad \varphi_{1}, \varphi_{2} \rightarrow \infty .
$$




\subsection{The Embedding $\mathrm{SO}(2) \subset \mathrm{SO}^{+}(2,1)$}

It is clear that $\mathfrak{s o}(2) \subset \mathfrak{s o}(2,1)$ as the maximal compact subalgebra. The embedding is quite visible from the particular form of the matrix $\mathcal{A}$ - one restricts to the skew-symmetric block by setting $x_{1}=x_{2}=0$ and ends up with a generator of rotation about $O Z$. Obviously the same relation holds for the corresponding groups and one can make it explicit by considering the power series expansion for $\exp \mathcal{A}$ in this case - the upper-left $2 \times 2$-block of $\mathcal{A}$ when squared gives minus identity and thus, for this particular choice of vector-parameter, we end up with the classical Rodrigues' formula

$$
\mathcal{R}(\hat{\mathbf{n}}, \varphi)=\cos \varphi \mathcal{I}+(1-\cos \varphi) \hat{\mathbf{n}} \otimes \hat{\mathbf{n}}^{t}+\sin \varphi \hat{\mathbf{n}}^{\times}, \quad \hat{\mathbf{n}}=\hat{\mathbf{e}}_{z}
$$

or, alternatively, one may use the basis of complex eigenvectors of $\mathcal{A}$ to derive

$$
\exp \mathcal{A}=\operatorname{diag}\left(\mathrm{e}^{\mathrm{i} \varphi}, \mathrm{e}^{-\mathrm{i} \varphi}, 1\right), \quad \mathbf{x} \rightarrow(0,0, \varphi)
$$

which is an equivalent $\mathrm{U}(1)$ representation.

For the physical applications considered in this paper, however, we are interested in the way ordinary $\mathrm{SO}(2)$ rotations interact with pure Lorentz boosts. Suppose we want to compose two $\mathrm{SO}^{+}(2,1)$ transformations - one of the former and one of the latter type, so that we have $\hat{\mathbf{c}}_{1} \in \mathbb{R}^{2}, \quad \hat{\mathbf{c}}_{2}=\hat{\mathbf{e}}_{z}$. Since the two vector parameters will be orthogonal in the hyperbolic (as well as in the Euclidean) metric, we have for the composition

$$
\left\langle\mathbf{c}_{2}, \mathbf{c}_{1}\right\rangle=\mathbf{c}_{2}+\mathbf{c}_{1}+\mathbf{c}_{2} \curlywedge \mathbf{c}_{1}
$$

and since the last vector on the right has a vanishing third component, we may write $\mathbf{c}_{2} \times \mathbf{c}_{1}$ instead of $\mathbf{c}_{2} \curlywedge \mathbf{c}_{1}$. Furthermore we may decompose $\hat{\mathbf{c}}_{1}=\cos \gamma \hat{\mathbf{e}}_{x}+\sin \gamma \hat{\mathbf{e}}_{y}$, where $\gamma$ is the polar angle, representing the unit vector $\hat{\mathbf{c}}_{1}$ in the $X O Y$-plane, and then use $\hat{\mathbf{e}}_{z} \times \hat{\mathbf{e}}_{x}=\hat{\mathbf{e}}_{y}, \hat{\mathbf{e}}_{z} \times \hat{\mathbf{e}}_{y}=-\hat{\mathbf{e}}_{x}$ to obtain

$$
\left\langle\mathbf{c}_{2}, \mathbf{c}_{1}\right\rangle=\tau_{1}\left(\cos \gamma-\tau_{2} \sin \gamma\right) \hat{\mathbf{e}}_{x}+\tau_{1}\left(\sin \gamma+\tau_{2} \cos \gamma\right) \hat{\mathbf{e}}_{y}+\tau_{2} \hat{\mathbf{e}}_{z} .
$$

Alternatively, we may compose in the reverse order

$$
\left\langle\mathbf{c}_{1}, \mathbf{c}_{2}\right\rangle=\tau_{1}\left(\cos \gamma+\tau_{2} \sin \gamma\right) \hat{\mathbf{e}}_{x}+\tau_{1}\left(\sin \gamma-\tau_{2} \cos \gamma\right) \hat{\mathbf{e}}_{y}+\tau_{2} \hat{\mathbf{e}}_{z}
$$

\section{Wigner Rotation}

A well-known fact from special relativity is that the composition of two noncollinear Lorentz boosts results in transformation that is no longer a pure boost, 
but can be expressed as a product of a rotation and a boost

$$
\mathcal{R}_{h}\left(\mathbf{c}_{2}\right) \mathcal{R}_{h}\left(\mathbf{c}_{1}\right)=\mathcal{R}_{h}\left(\tilde{\tau} \mathbf{e}_{z}\right) \mathcal{R}_{h}(\mathbf{c})
$$

where the first factor on the righthand side stands for the rotational contribution (a pure rotation in $\mathrm{SO}^{+}(2,1)$ is possible only about the third axis $\left.\mathbf{e}_{z}\right)$ and the second one is the hyperbolic (boost) contribution to the result.

Since by construction $\mathbf{c}_{1}$ and $\mathbf{c}_{2}$ have vanishing third components, they can be expressed with planar vectors, or more conveniently - complex numbers $z_{1}, z_{2} \in \mathbb{C}$

$$
z_{k}=x_{k}+\mathrm{i} y_{k} \rightarrow \mathbf{c}_{k}=\left(x_{k}, y_{k}, 0\right)^{t}, \quad k=1,2 .
$$

From (61) we see that the third component of the composition $\left\langle\mathbf{c}_{2}, \mathbf{c}_{1}\right\rangle$ is responsible for the rotational contribution to the compound transformation. Since $\mathbf{c}_{1}$ and $\mathbf{c}_{2}$ lie in the $X O Y$-plane, this component is quite easy to obtain, using (22)

$$
\tilde{\tau}=\left\langle\mathbf{c}_{2}, \mathbf{c}_{1}\right\rangle_{z}=\frac{x_{1} y_{2}-x_{2} y_{1}}{1+x_{1} x_{2}+y_{1} y_{2}}=-\frac{\Im\left(1+z_{1} \bar{z}_{2}\right)}{\Re\left(1+z_{1} \bar{z}_{2}\right)}
$$

and therefore we have for the angle of that rotation $\theta=2 \arctan \tilde{\tau}$ the simple expression

$$
\theta=-2 \arg \left(1+z_{1} \bar{z}_{2}\right) .
$$

The latter is known as Wigner angle and plays an important role in both special relativity and quantum mechanics. The simple and compact formula we obtain is very convenient for the interpretation of $\theta$ as a Berry phase [6,25], or (negative) area of a hyperbolic triangle [2].

Note that if we reverse the order in the composition, only the sign of $\theta$ is changed. Moreover, once we have determined $\tilde{\tau}$, it is not difficult to retrieve the vector parameter $\mathbf{c}$ of the boost in the decomposition

$$
\left\langle\mathbf{c}_{2}, \mathbf{c}_{1}\right\rangle=\left\langle\tilde{\tau} \mathbf{e}_{z}, \mathbf{c}\right\rangle .
$$

With the help of (61) and (62) it is almost straightforward to write

$$
\mathbf{c}_{ \pm}=\frac{\Re\left(1+z_{1} \bar{z}_{2}\right)}{\left|1+z_{1} \bar{z}_{2}\right|^{2}}\left(\begin{array}{c}
\Re\left(z_{12}\right) \pm \tilde{\tau} \Im\left(z_{12}\right) \\
\Im\left(z_{12}\right) \mp \tilde{\tau} \Re\left(z_{12}\right) \\
0
\end{array}\right), \quad z_{12}=z_{1}+z_{2}
$$


where the two solutions $\mathbf{c}_{+}$and $\mathbf{c}_{-}$correspond to the forward $\left\langle\mathbf{c}_{2}, \mathbf{c}_{1}\right\rangle$ and backward $\left\langle\mathbf{c}_{1}, \mathbf{c}_{2}\right\rangle$ composition respectively. Note that we may easily find the components of the corresponding pseudo-quaternion $\zeta$ with the help of (19)

$$
\begin{aligned}
\mathbf{c}_{ \pm}{ }^{2} & =\left|\frac{z_{1}+z_{2}}{1+z_{1} \bar{z}_{2}}\right|^{2} \Longrightarrow \zeta_{0}=\left(1+\mathbf{c}_{ \pm}^{2}\right)^{-\frac{1}{2}}=\frac{\left|1+z_{1} \bar{z}_{2}\right|}{\sqrt{\left|1+z_{1} \bar{z}_{2}\right|^{2}-\left|z_{1}+z_{2}\right|^{2}}} \\
\boldsymbol{\zeta}_{ \pm} & =\frac{\Re\left(1+z_{1} \bar{z}_{2}\right)}{\sqrt{\left|1+z_{1} \bar{z}_{2}\right|^{2}-\left|z_{1}+z_{2}\right|^{2}}}\left(\begin{array}{c}
\Re\left(z_{1}+z_{2}\right) \pm \tilde{\tau} \Im\left(z_{1}+z_{2}\right) \\
\Im\left(z_{1}+z_{2}\right) \mp \tilde{\tau} \Re\left(z_{1}+z_{2}\right) \\
0
\end{array}\right)
\end{aligned}
$$

where we have written only the solution with positive scalar part, since $\zeta$ and $-\zeta$ participate symmetrically (the \pm subscript stands for the different permutations of the two boosts).

In scattering theory we encounter a $\mathrm{SU}(1,1)$ representation in the monodromy matrix formalism. More precisely, the monodromy matrix $\mathcal{M}$ relates the left and right free particle asymptotic solutions (or rather one-jets of solutions) in the one dimensional scattering problem. When we write these solutions in a real basis (with sin and cos functions), this matrix is bound to be $\operatorname{SL}(2, \mathbb{R})$, due to a conservation law for the phase volume. Then, we conclude that back in the standard complex basis, usually preferred in quantum mechanics, $\mathcal{M} \in \mathrm{SU}(1,1)$. Whether the process takes place at a point or over an interval, the monodromy matrix (as an alternative to the scattering matrix) describes the scattering potential and its entries are given by the corresponding transition and reflection coefficients. For simplicity of notations we are going to write it as

$$
\mathcal{M}=\left(\begin{array}{cc}
\alpha & \beta \\
\bar{\beta} & \bar{\alpha}
\end{array}\right) \in \operatorname{SU}(1,1)
$$

so if we have two scatterers on the same axis with potentials $V_{1}$ and $V_{2}$, the compound monodromy matrix will be given by $\mathcal{M}=\mathcal{M}_{2} \mathcal{M}_{1}$. It would be convenient to expand these matrices in the basis (1) built in Section 2, which is equivalent to associating to each of them a pseudo-quaternion: $\mathcal{M}_{1} \rightarrow \xi, \mathcal{M}_{2} \rightarrow \zeta$ and $\mathcal{M} \rightarrow \zeta \xi$, and then use the composition law (21) to find $\zeta$ explicitly. We may also use complex coordinates $\alpha_{i}, \beta_{i}$ to write $\mathcal{M}_{i}$ in the form (70). Note that if we project this construction (dividing by the scalar part), we would obtain the composition for the vector-parameters $(22)$ in the corresponding $\mathrm{SO}^{+}(2,1)$ representation.

What we are aiming for is to find the angle of Wigner rotation that appears as a result of such superposition of scatterers. Mathematically, this process is explained 
with the fact that the compound monodromy $\mathcal{M}$ can be represented as a composition of an elliptic and hyperbolic transformation

$$
\mathcal{M}=\mathcal{M}_{e} \mathcal{M}_{h}, \quad \mathcal{M}_{e} \in \mathrm{SO}(2, \mathbb{R}) .
$$

In particular we may also represent $\mathcal{M}_{e}$ and $\mathcal{M}_{h}$ with pseudo-quaternions, say $\tilde{\zeta}$ and $\tilde{\xi}$ respectively, where $\tilde{\xi}$ has vanishing fourth component while for $\tilde{\zeta}$ - the second and the third are zero. If we map these to $\mathrm{SO}^{+}(2,1)$ via projection, the composition for the vector parameters coincides with (61) so we may find the Wigner angle from the third component of the compound parameter as

$$
\theta=2 \arctan \left\langle\mathbf{c}_{2}, \mathbf{c}_{1}\right\rangle_{z}
$$

and since $\zeta \xi=\tilde{\zeta} \tilde{\xi}$, lifting up back to the universal cover gives simply

$$
\left\langle\mathbf{c}_{2}, \mathbf{c}_{1}\right\rangle=\frac{\zeta_{0} \boldsymbol{\xi}+\xi_{0} \zeta+\zeta \curlywedge \boldsymbol{\xi}}{\zeta_{0} \xi_{0}+\zeta \cdot \boldsymbol{\xi}} .
$$

The normalized $z$-component is given by $\frac{\zeta_{3}}{\zeta_{0}}$, that is the ratio between the imaginary and the real part of the first matrix entry of the composition $\mathcal{M}_{2} \mathcal{M}_{1}$ if we work in complex coordinates, i.e.,

$$
\theta=2 \arg \left(\alpha_{2} \alpha_{1}+\beta_{2} \bar{\beta}_{1}\right), \quad \alpha_{1}, \alpha_{2} \in \mathbb{R}
$$

in a complete correspondence with (66) (in order to see this one needs only to introduce $z_{k}=\frac{\beta_{k}}{\alpha_{k}}$ ). The restriction on the $\alpha_{k}$ 's guarantees that the $\mathcal{M}_{k}$ 's represent pure boosts. Moreover, one may easily find the remaining boost in the decomposition, substituting $z_{k}=\frac{\beta_{k}}{\alpha_{k}}$ in (69)

$$
\zeta_{0}=\frac{|\delta|}{\sqrt{|\delta|^{2}-|\nu|^{2}}}, \quad \boldsymbol{\zeta}_{ \pm}=\frac{\Re(\delta)}{\left|\alpha_{1} \alpha_{2}\right| \sqrt{|\delta|^{2}-|\nu|^{2}}}\left(\begin{array}{c}
\Re(\nu) \pm \tilde{\tau} \Im(\nu) \\
\Im(\nu) \mp \tilde{\tau} \Re(\nu) \\
0
\end{array}\right)
$$

where we use the short notations $\delta=\alpha_{1} \alpha_{2}+\beta_{1} \bar{\beta}_{2}$ and $\nu=\alpha_{1} \beta_{2}+\beta_{1} \alpha_{2}$.

Now to replace $\alpha_{i}$ and $\beta_{i}$ with the physical quantities $t_{i}$ and $r_{i}$ - the transition and reflection coefficients (the latter is usually denoted by $r_{+}$in the literature), defined 
by the asymptotic behavior of the solutions

$$
\begin{aligned}
& \Psi(k, x) \sim \mathrm{e}^{\mathrm{i} k x}+r(k) \mathrm{e}^{-\mathrm{i} k x}, \quad x \rightarrow-\infty \\
& \Psi(k, x) \sim t(k) \mathrm{e}^{\mathrm{i} k x}, \quad x \rightarrow \infty .
\end{aligned}
$$

Using the well-known relations (see $[3,14,15]$ )

$$
\alpha=\frac{1}{\bar{t}}, \quad \beta=-\frac{\bar{r}}{\bar{t}}
$$

and restricting to $\mathrm{SU}(1,1)$ matrices with real diagonal entries $t_{1}, t_{2} \in \mathbb{R}$ as they are the ones corresponding to pure boosts, we end up with the compact expression

$$
\theta^{ \pm}= \pm 2 \arg \left(1+r_{1} \bar{r}_{2}\right)
$$

for the forward and the backward composition respectively, and the condition for zero Wigner rotation in both cases has the form $r_{1} \bar{r}_{2} \in \mathbb{R}$.

The matrix of the remaining boost in this representation can be derived from (69) with $z_{k}=-\bar{r}_{k}$. In in pseudo-quaternion coordinates it has the form

$$
\begin{aligned}
\mathbf{c}_{ \pm}^{2} & =\left|\frac{r_{1}+r_{2}}{1+r_{1} \bar{r}_{2}}\right|^{2} \Longrightarrow \zeta_{0}=\left(1+\mathbf{c}_{ \pm}^{2}\right)^{-\frac{1}{2}}=\frac{\left|1+r_{1} \bar{r}_{2}\right|}{\sqrt{\left|1+r_{1} \bar{r}_{2}\right|^{2}-\left|r_{1}+r_{2}\right|^{2}}} \\
\boldsymbol{\zeta}_{ \pm} & =\frac{\Re\left(1+r_{1} \bar{r}_{2}\right)}{\sqrt{\left|1+r_{1} \bar{r}_{2}\right|^{2}-\left|r_{1}+r_{2}\right|^{2}}}\left(\begin{array}{c}
-\Re\left(r_{1}+r_{2}\right) \pm \tilde{\tau} \Im\left(r_{1}+r_{2}\right) \\
\Im\left(r_{1}+r_{2}\right) \pm \tilde{\tau} \Re\left(r_{1}+r_{2}\right) \\
0
\end{array}\right)
\end{aligned}
$$

respectively for the forward and the backward composition of scatterers.

With a little effort we can relate the above results to the Thomas precession phenomenon, considering composition of infinitesimal vector parameters. However, we chose to ignore this opportunity for now and only refer to $[4,28]$ for different, but related treatment of the problem.

\section{Concluding Remarks}

In the present article we focus mainly on the generalized Euler parameters in the decomposition of three-dimensional pseudo-rotations. Using pseudo-quaternion and vector-parameter techniques, we manage to obtain quite simple and compact 
explicit solutions, similar to the ones we give for the Euclidean (pure rotational) case [5].

Due to the well-known Lie algebra isomorphisms $\mathfrak{s o}(2,1) \simeq \mathfrak{s u}(1,1) \simeq \mathfrak{s l}(2, \mathbb{R})$, we are able to give analogous relations for the structure groups of the upper halfplane and the Poincaré disk - actually, projecting and lifting up to the universal cover is so easy for the formulae we obtain, that we did not bother to write all the expressions.

Finally, we provide a well-known physical example - calculation of the Wigner angle in special relativity and scattering theory, which appears to be particularly easy using the machinery developed here.

Since most of the work is concerned with the three-dimensional Lorentz group $\mathrm{SO}^{+}(2,1)$, we note that it has plenty of physical applications and should not be considered a mere toy model for $3+1$ dimensional special relativity. This has become particularly apparent since the breakthrough in graphenes [7].

However, the $3+1$ dimensional case seems like a natural extension and can be treated with s imilar techniques. Another investigation of physical relevance would be considering the case of coordinate frame, attached to the moving object. Actually, most of the results, obtained here, maintain their validity with a slight modification, but we reserve the pleasure of describing this correspondence for future work.

\section{References}

[1] Ahlfors L., Möbius Transformations in Several Dimensions, School of Mathematics, University of Minessota 1981.

[2] Aravind P., The Wigner Angle as an Anholonomy in Rapidity Space, Am. J. Phys. 65 (1997) 634-636.

[3] Arnold V., Geometrical Methods in the Theory of Ordinary Differential Equations, Springer, New York 1983.

[4] Ben-Manahem A., Wigner's Rotation Revisited, Am. J. Phys. 53 (1985) 6266.

[5] Brezov D., Mladenova C. and Mladenov I., Vector Decompositions of Rotations, J. Geom. Symmetry Phys. 28 (2012) 67-103.

[6] Bruno P., The Berry Phase in Magnetism and the Anomalous Hall Effect, Handbook of Magnetism and Advanced Magnetic Materials vol. 1, Wiley, Chichester (2007), pp 540-558.

[7] Castro N., Guinea F., Peres N., Novoselov K. and Geim A., Electronic Properties of Graphenes, Rev. Mod. Phys. 81 (2009) 109-162. 
[8] Chub V., On the Possibility of Application of One System of Hypercomplex Numbers in Inertial Navigation, Mech. Solids 37 (2002) 1-17.

[9] Davenport P., Rotations About Nonorthogonal Axes, AIAA Journal 11 (1973) 853-857.

[10] Fedorov F., The Lorentz Group (in Russian), Science, Moskow 1979.

[11] Gibbs J., Elements of Vector Analisys, Tuttle, Morehouse \& Taylor, New Haven 1881.

[12] Gilmore R., Relations Among Low-dimensional Simple Lie Groups, J. Geom. Symmetry Phys. 28 (2012) 1-45.

[13] Helgason S., Differential Geometry, Lie Groups and Symmetric Spaces, Academic Press, San Diego 1978.

[14] Hurt N., Geometric Quantization in Action, Reidel, Dordrecht 1983.

[15] Kandilarov B. and Detcheva V., Transfer-Matrix Approach and Basic Ideas of the Zone Theory (in Bulgarian), Bulgarian Academy of Sciences, Sofia 1988.

[16] Kocik J., Clifford Algebras and Euclid's Parameterization of Pythagorean Triples, Adv. Appl. Clifford Algebras 17 (2007) 71-93.

[17] Kuipers J., Quaternions and Rotation Sequences, Geometry, Integrability and Quantization 1 (2000) 127-143.

[18] Lee, H. On Plane Factorizations of Pseudo-Euclidean Rotations, Quart. J. Math. 15 (1944) 7-10.

[19] Mladenov I., Geometry of the Saxon-Hutner Theorem, Phys. Lett. A 230 (1997) 245-252.

[20] Mladenov I., On the Saxon-Hutner Theorem, C. R. Bulg. Acad. Sci. 38 (1985) 993-996.

[21] Mladenova C. and Mladenov I., Vector Decomposition of Finite Rotations, Rep. Math. Phys. 68 (2011) 107-117.

[22] Mladenov I. and Vassileva J., A Note on Hibridization, Commun. Math. Chem. 11 (1981) 69-73.

[23] Mladenova C., Group Theory in the Problems of Modeling and Control of Multi-Body Systems, J. Geom. Symmetry Phys. 8 (2006) 17-121.

[24] Mladenova C., An Approach to Description of a Rigid Body Motion, C. R. Acad. Sci. Bulg. 38 (1985) 1657-1660.

[25] Mukunda N., Aravind P. and Simon R., Wigner Rotations, Bargmann Invariants and Geometric Phases, J. Phys. A: Math. \& Gen. 36 (2003) 2347-2370

[26] Palais B., Palais R. and Rodi S., A Disorienting Look at Euler's Theorem on the Axis of a Rotation, Am. Math. Monthly 116 (2009) 892-909. 
[27] Piovan G. and Bullo F., On Coordinate-Free Rotation Decomposition Euler Angles About Arbitrary Axes, IEEE Trans. Robotics 28 (2012) 728-733.

[28] Rhodes A. and Semon M., Relativistic Velocity Space, Wigner Rotation and Thomas Precession, Am. J. Phys. 72 (2004) 943-961

[29] Rodrigues O., Des lois géométriques qui regissent les déplacéments d'un systéme solide dans l'espace, et de la variation des coordonnées provenant de ces déplacéments considérés indépendamment des causes qui peuvent les produire, J. Math. Pures Appl. 5 (1840) 380-440.

[30] Ungar A., Analytic Hyperbolic Geometry: Mathematical Foundations and Applications, World Scientific, Singapore (2005)

[31] Ungar A., The Proper-Time Lorentz Group Demystified, J. Geom. Symmetry Phys. 4 (2005) 69-95.

[32] Ward J., Quaternions and Cayley Numbers: Algebra and Applications, Kluwer, Dordrecht 1997.

[33] Wittenburg J., Dynamics of Multibody Systems, Springer, Berlin 2008.

[34] Wohlhart K., Decomposition of a Finite Rotation into Three Consecutive Rotations About Given Axes, In: Proc. VI-th Int Conf. on Theory of Machines and Mechanisms, Czechoslovakia, Liberec 1992, pp 325-332.

Danail S. Brezov

University of Architecture

Civil Engineering and Geodesy

1 Hristo Smirnenski Blvd.

1046 Sofia, Bulgaria

E-mail address: danail.brezov@gmail.com

Clementina D. Mladenova

Institute of Mechanics

Bulgarian Academy of Sciences

Acad. G. Bonchev Str., Bl. 4

1113 Sofia, Bulgaria

E-mail address: cleme imbm.bas.bg

Ivaïlo M. Mladenov

Institute of Biophysics

Bulgarian Academy of Sciences

Acad. G. Bonchev Str., Bl. 21

1113 Sofia, Bulgaria

E-mail address: mladenov@bio21.bas.bg 